\title{
Silicon spin diffusion transistor: materials, physics and device characteristics
}

\author{
C.L. Dennis, C.V. Tiusan, J.F. Gregg, G.J. Ensell and S.M. Thompson
}

\begin{abstract}
The realisation that everyday electronics has ignored the spin of the carrier in favour of its charge is the foundation of the field of spintronics. Starting with simple two-terminal devices based on giant magnetoresistance and tunnel magnetoresistance, the technology has advanced to consider three-terminal devices that aim to combine spin sensitivity with a high current gain and a large current output. These devices require both efficient spin injection and semiconductor fabrication. In the paper, a discussion is presented of the design, operation and characteristics of the only spin transistor that has yielded a current gain greater than one in combination with reasonable output currents.
\end{abstract}

\section{Introduction}

Everyday electronic devices manipulate carriers solely based on their charge, either positive or negative. Their operation ignores the fact that those carriers also have a spin, either spin up or spin down. Spintronics aims to remedy this deficiency by manipulating the spin as well as the charge of the carrier in nanoscale devices. Spintronics goes beyond the simple use of magnetic fields to alter the movement of charge, as in the Hall effect. Instead, spintronics determines and/or senses the spin orientation of the carriers by incorporating magnetic materials into conventional devices. Spins injected into a material are polarised either optically or by passing through a magnetic material, which polarises the carriers parallel (or antiparallel depending on the material) to the direction of magnetisation. The difference in behaviour of carriers of different spin types only becomes apparent when they travel through a magnetic material. For example, when spin-up electrons are injected into a magnetic material with the net magnetisation pointing in the same direction, these spins pass through relatively unscathed. However, spin-down electrons are heavily scattered. This leads to different conductivities (resistivities) for the different spin types. It is upon this principle that most of the suggested devices are based.

This field has expanded rapidly in recent years. It was only a few years from the discovery of giant magnetoresistance (GMR) [1] to the first commercial production of a GMR-based read head for a disk drive (by IBM in 1997). Recent research has focused on expanding the

\footnotetext{
(C) IEE, 2005
}

IEE Proceedings online no. 20050008

doi:10.1049/ip-cds:20050008

Paper first received 10th January and in revised form 1st April 2005

C.L. Dennis and J.F. Gregg are with the Clarendon Laboratory, Parks Road, Oxford OX1 3PU, UK

C.V. Tiusan is with the Laboratoire de Physique des Materiaux, Faculté des Sciences, Université H. Poincaré, Nancy 1, Boulevard des Aiguillettes B.P. 239 54506 Vandoeuvre les Nancy, France

G.J. Ensell is with the Department of Electronics, University of Southampton, Southampton SO17 1BJ, UK

S.M. Thompson is with the Department of Physics, University of York, York, YO10 5DD, UK

E-mail: c.dennis1@physics.ox.ac.uk scope of spintronics from two-terminal to three-terminal devices. This has led to the development of a number of different three-terminal designs [2-9] (which will be briefly discussed in Section 2) that aim to exploit the spin-dependent scattering of charge carriers to yield a device with high current gain and high magnetic sensitivity. This paper will focus on the silicon-based spin diffusion transistor, the only spin transistor which yields a current gain greater than one. The discussion will begin with the fabrication, followed by the experimental results. It will conclude with a discussion of how the fabrication and materials affect the output characteristics.

\section{General operating principles of spin transistors}

\subsection{Johnson transistor}

The first spin transistor was the Johnson bipolar transistor [2], which added a third terminal connection to the nonmagnetic spacer layer in a CPP-GMR trilayer (see Fig. 1). Bipolar has double meaning: positive and negative charge carriers, up- and down-spin carriers, and output which is either a positive or negative current/voltage. This spin transistor device requires that the thickness of the layers be comparable to or smaller than the spin diffusion length of the material.

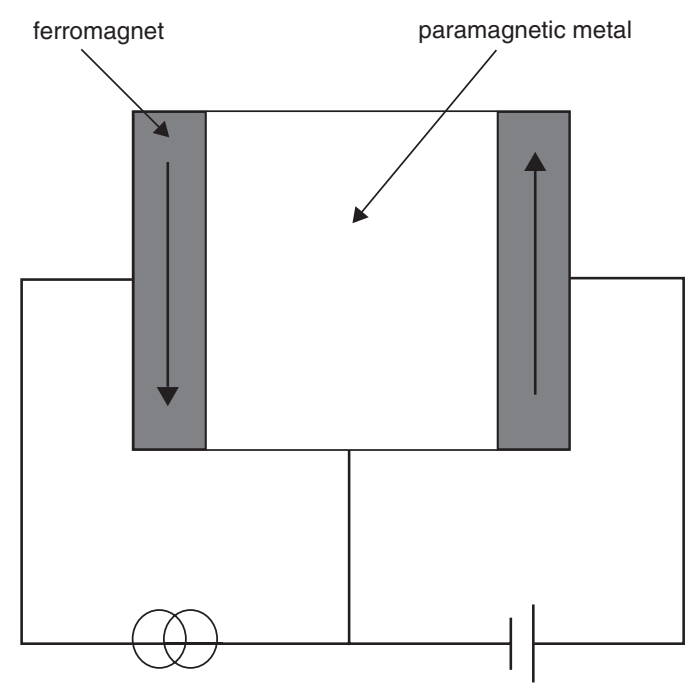

Fig. 1 Schematic of the Johnson bipolar transistor 
As the electrical characteristics of this purely ohmic device are magnetically tunable, it can potentially be used as a field sensor or as nonvolatile magnetic random access memory. However, owing to its all-metal construction, its operation yields only small voltage output changes and no power or current gain (power gain may be possible in 5-terminal (as opposed to a 3-terminal) architecture). If such a device could generate a current gain, it could potentially be used to make logic devices [10].

\subsection{Monsma transistor}

The next step was to try and incorporate semiconductors with magnetism to further expand the field of spintronics by generating novel functionality. The Monsma transistor [3], produced at the University of Twente, was the first hybrid spintronic device (see Fig. 2). (The Mizushima MIFS transistor [4] is a second variant on this design, where one of the Schottky barriers is replaced by a tunnel barrier.) First fabricated in 1995, it sandwiched a CPP-GMR multilayer between two semiconductors (silicon). Schottky barriers form at the interfaces between the silicon and the metal structure and these absorb the bias voltages applied between pairs of terminals. The collector Schottky barrier is back-biased and the emitter Schottky is forward-biased. This injects (unpolarised) hot electrons from the semiconductor emitter into the metallic base high above its Fermi energy. If the hot electrons travel across the thickness of the base and retain enough energy to surmount the collector Schottky barrier, then they will exit through the collector; otherwise, they will exit via the base.
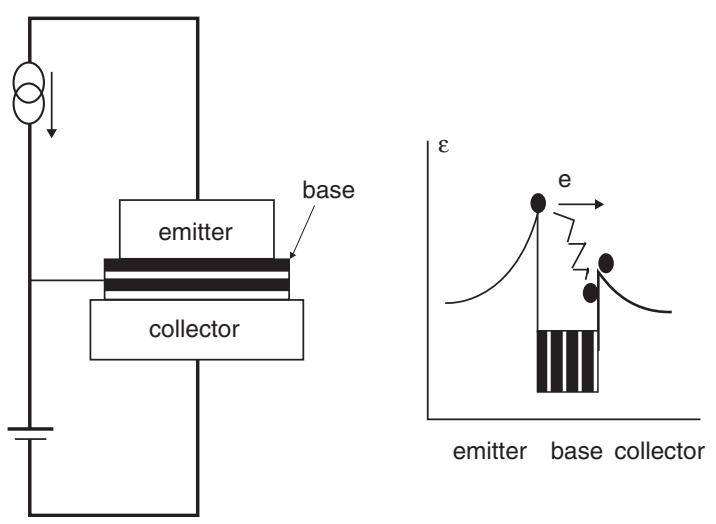

Fig. 2 Schematic of the Monsma transistor (from [3])

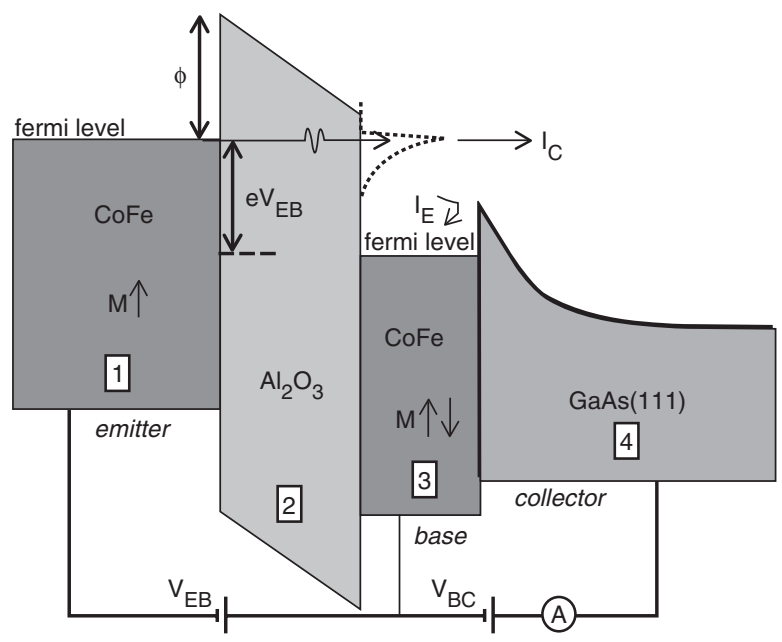

Fig. 3 Schematic of the magnetic tunnel transistor (from [5])
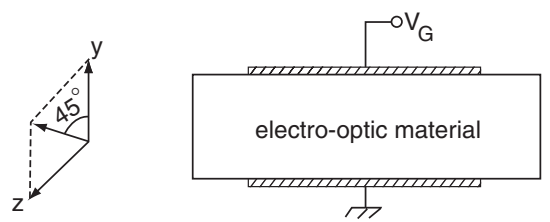

polariser

H7

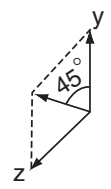

analyser

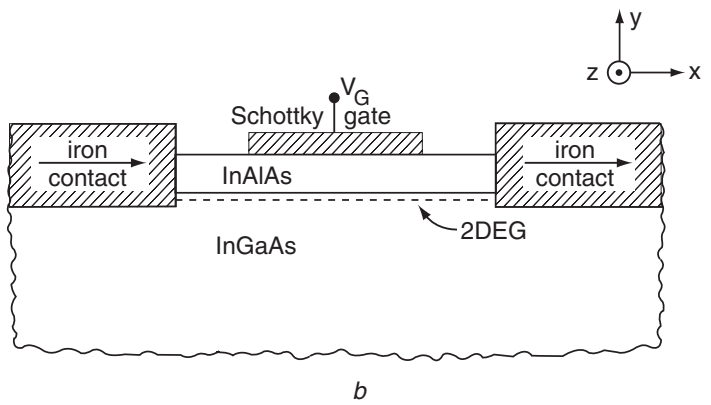

Fig. 4 Schematic of the spin FET (from [7])

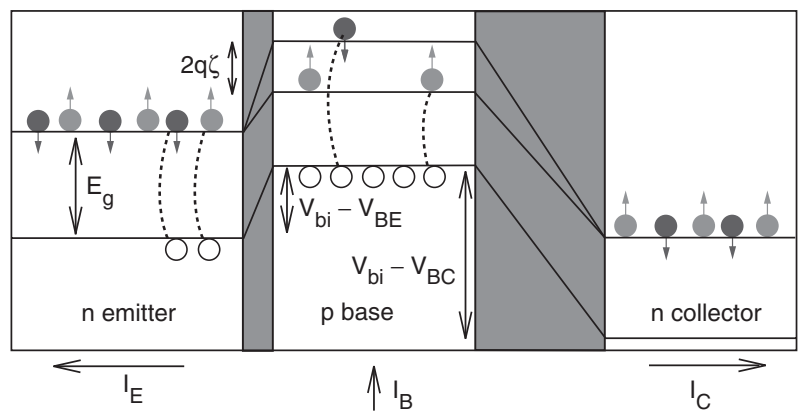

Fig. 5 Schematic of the magnetic bipolar transistor (from [8])

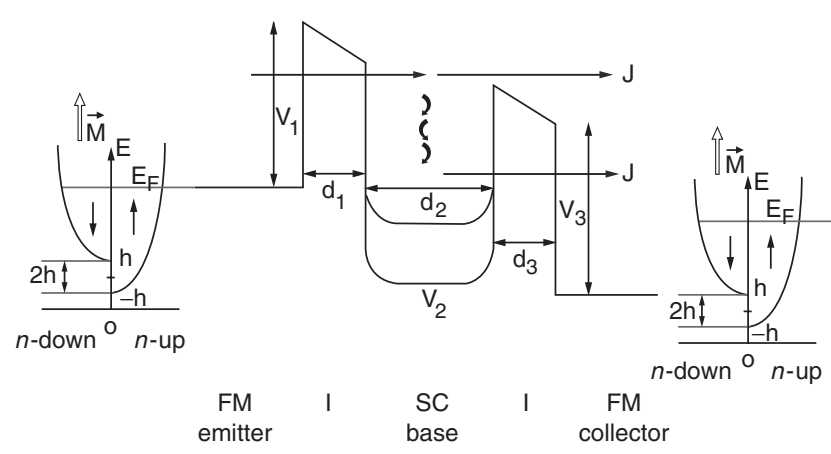

Fig. 6 Band structure of the spin diffusion transistor

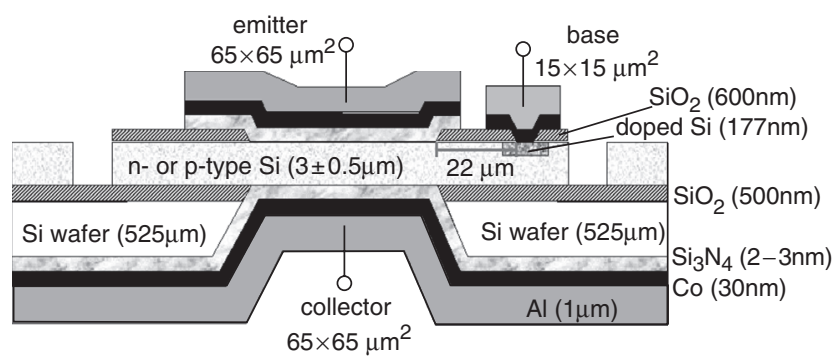

Fig. 7 Structural schematic diagram of the fabricated spin diffusion transistor with silicon base

The collector-emitter separation is $2.2 \mu \mathrm{m}$ and the emitter-base separation is $22 \mu \mathrm{m}$. Note that the collector and emitter contacts are metal-insulator-semiconductor junctions and the base contact is ohmic (metal-semiconductor junction). All three contacts have a thin film of cobalt, but the base cobalt film is present simply for ease of fabrication 
By varying the magnetic configuration of the base magnetic multilayer the operator can determine how much energy the hot electrons lose in their passage across the base. If the magnetic layers are antiferromagnetically aligned in the multilayer, then both spin types experience heavy scattering in one of the magnetic layer orientations, so the average energy of both spin types as a function of distance into the base follows an exponential decay curve. On the other hand, if the magnetic multilayer is in an applied field and its layers are all aligned, one spin type gets scattered heavily in every magnetic layer, whereas the other travels through the structure relatively unscathed. It may thus be seen that, for parallel magnetic alignment, spins with higher average energy impinge on the collector barrier and the collected current is correspondingly higher.

However, one limiting factor of this device is that it only used the semiconductor to control the distribution of applied potentials across the device; it is the metallic components that are spin selective. Despite this, the Monsma transistor represents a very important step in the evolution of spintronics. It has electrical characteristics that are magnetically tunable, plus a current gain and magnetic sensitivity that are sufficiently large so that, with help from some conventional electronics, it is a candidate for a practical working device. However, to release the full potential of hybrid spintronics, the devices need to exploit spin-dependent transport in the semiconductor itself.

\subsection{Magnetic tunnel transistor (MTT)}

The next generation of spin transistor devices modified the Monsma transistor by incorporating the knowledge gained from the theoretical analysis of spin injection. The Schottky barriers were replaced by tunnel barriers to form the magnetic tunnel transistor [5]. This device (shown in Fig. 3) is fabricated by depositing a magnetic tunnel junction on top of a GaAs substrate. The first ferromagnet acts as the emitter, the second ferromagnet as the base, and the
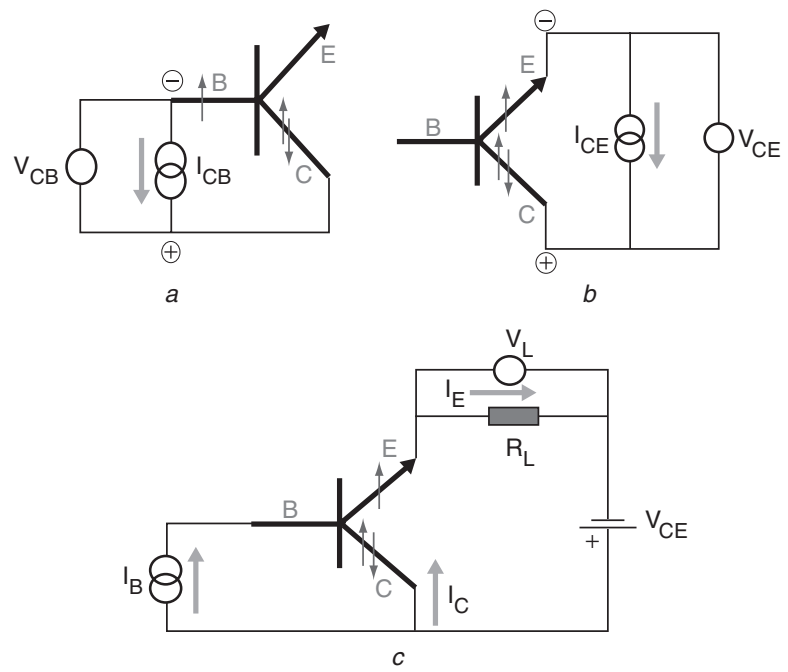

Fig. 8 Measurement circuit for the collector to base circuit, the collector to emitter circuit, and common-collector configuration The collector, base and emitter are labelled C, B and E, respectively. The grey arrows indicate sign conventions for positive current. A black single arrow indicates that the magnetic layer on that contact is fixed, whereas the black double arrows indicate that the magnetisation of that contact is free. The + and - signs indicate the polarity of the applied voltage. Note that the sign conventions are the same regardless of whether the Si base is p-type or n-type

$a$ Collector to base circuit

$b$ Collector to emitter circuit

$c$ Common-collector configuration semiconductor acts as the collector. The device works by injecting spin-polarised electrons across the tunnel barrier from the first ferromagnet into the second ferromagnet. The voltage applied between the emitter and collector is dropped across the tunnel barrier, thereby controlling the amount of current that reaches the base (the second ferromagnet). If the base is thin enough, then the current reaching the base will travel ballistically across the base and have enough energy to surmount the Schottky barrier between the second ferromagnetic metal and the semiconductor. (The ferromagnet/insulator/ferromagnet/insulator/metal (FIFIM) transistor by Ounadjela and Hehn [6] is a variant on this, where the Schottky barrier is replaced by another tunnel junction.) Any electrons that do not have enough energy will be swept out the base as a base current. The magnetic sensitivity again derives from the differential scattering of hot electrons of different spin types in the second magnetic layer. The sensitivity is dramatically 'amplified' by the exponential spin-energy decay; incidentally, this latter feature makes the magnetic trilayer structure on the left of the device a very effective spin polariser. However, it is again the metallic components that determine the magnetic sensitivity.
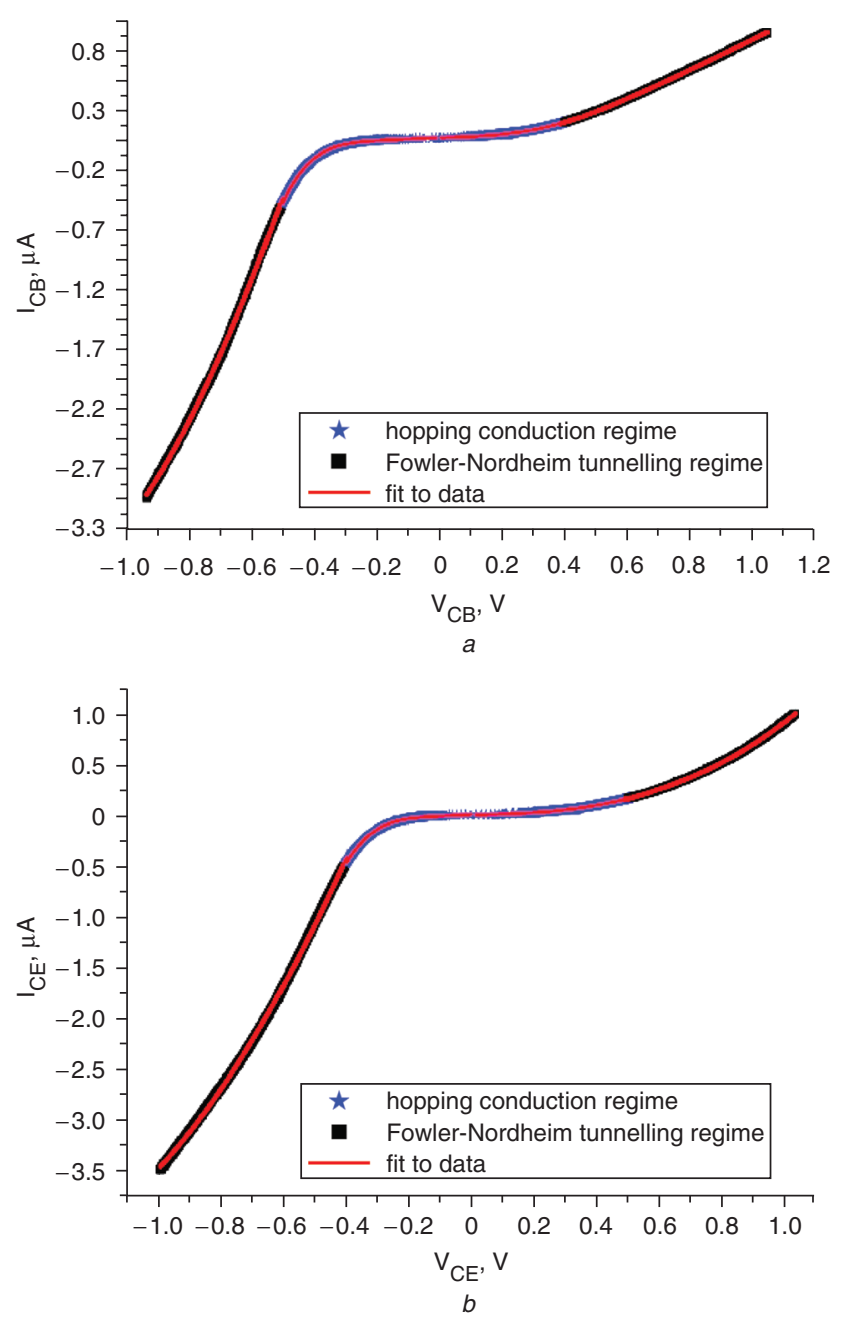

Fig. 9 Two-terminal characteristics of the p-type transistor The dots indicate measured data points (where black dots indicate the region where Fowler-Nordheim tunnelling dominates and blue dots indicate the region where hopping conduction dominates) and the solid red lines are the fits, according to the regime the data is in $a$ V-I curve for p-type spin transistor (II-6): collector to base, four regimes and their fits

$b \mathrm{~V}-\mathrm{I}$ curve for p-type spin transistor (II-6): collector to emitter, four regimes and their fits 

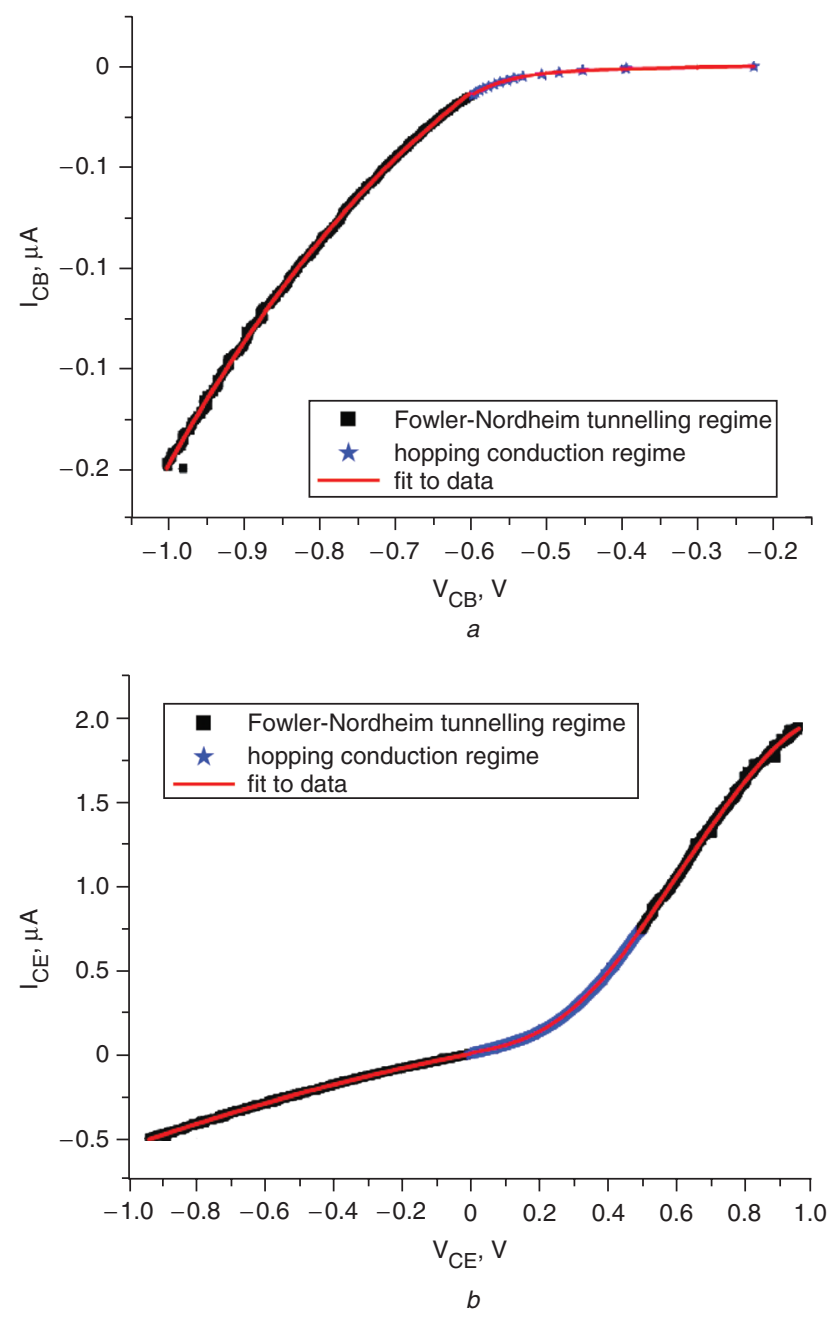

Fig. 10 Two-terminal characteristics of the n-type transistor The black dots indicate measured data points (where black dots indicate the region where Fowler-Nordheim tunnelling dominates and blue dots indicate the region where hopping conduction dominates) and the solid red lines are the fits, according to the regime the data is in $a \mathrm{~V}-\mathrm{I}$ curves for n-type spin transistor (II-8): collector to base, four regimes and their fits

$b \mathrm{~V}-\mathrm{I}$ curve for n-type spin transistor (II-8): collector to emitter, four regimes and their fits

\subsection{Spin field-effect transistor (spin-FET)}

A fourth transistor was developed in parallel with the previous devices: the experimental realisation of a spin-FET (see Fig. 4) based on the proposal by Datta and Das [7] in 1990. This transistor is a modification of a field-effect transistor (FET), where an applied electric field changes the width of the depletion region and, hence, its electrical resistance. In a spin FET, spin-polarised electrons are injected from a magnetic source into a semiconductor channel. During passage through the channel, these electrons undergo Rashba precession, the frequency of which depends on the gate voltage. Finally, the electrons are analysed by spin selective scattering in the magnetic drain. Hence, the actual electrical characteristics are dependent on, not only the magnetic orientation of the source and drain, but also on the gate voltage. Gardelis and co-workers [11] have made a step towards realising this device, but the gate functionality remains to be demonstrated. It may be noted that the characteristics of the spin FET differ from those of the device in the following subsection in that its $\mathrm{g}_{\mathrm{m}}$ may be a periodic function of gate voltage and may change sign on application of a magnetic field.
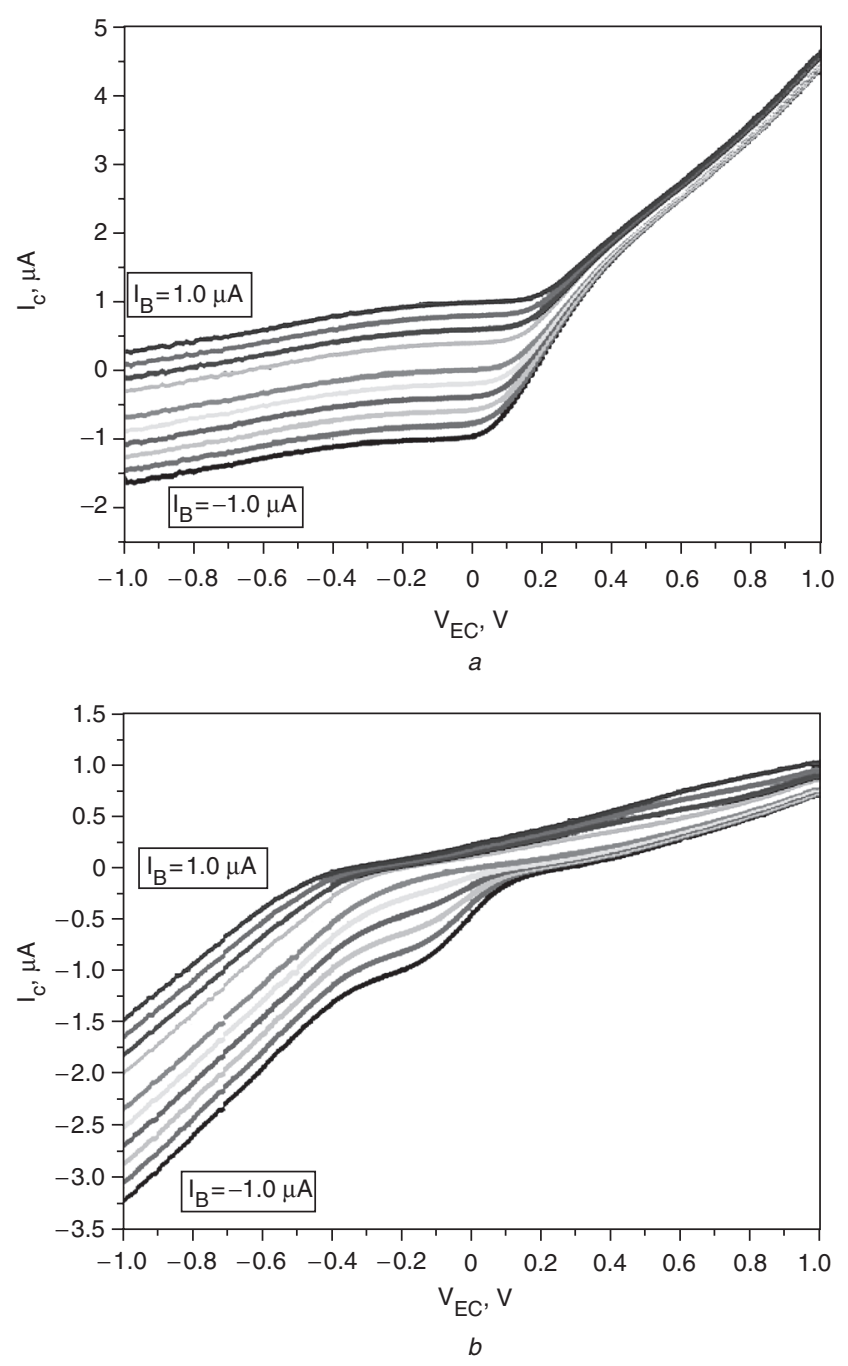

Fig. $11 I_{C}-V_{E C}$ characteristics of spin diffusion transistors in common-collector configuration and zero applied field

The load resistor $R_{L}=9976 \Omega$. The step size of $I_{B}$ is $0.2 \mu \mathrm{A}$ with the starting and ending currents as indicated in the boxes

a Common collector configuration of p-type Southampton spin transistor (II-6), $\mathrm{H}=0$ Oe, $\mathrm{R}_{\mathrm{L}}=9.976 \mathrm{k} \Omega$

$b$ Common collector configuration of n-type Southampton spin transistor (II-8), $\mathrm{H}=0 \mathrm{Oe}, \mathrm{R}_{\mathrm{L}}=9.976 \mathrm{k} \Omega$

\subsection{Magnetic bipolar transistor (MBT)}

This fifth variant, which is analogous to an ordinary bipolar junction transistor (BJT), has been explored theoretically in $[8,9]$. Like the spin FET, the magnetic bipolar transistor (see Fig. 5) has not yet been realised experimentally, although a prototype has been fabricated [12]. Like the BJT, this device consists of two $\mathrm{p}-\mathrm{n}(\mathrm{n}-\mathrm{p})$ junctions connected in series and operates in a similar manner. The active (current amplification) region occurs under the same conditions for both devices: the emitter-base junction is forward-biased and the base-collector junction is reversebiased. However, in the MBT, the emitter and collector are nonmagnetic, but the base is magnetic, creating spin-split conduction bands (see Fig. 5). It may also have a spinpolarised injector. As the conduction band in the base has a spin splitting $2 \mathrm{q} \zeta$, the electrons that flow from emitter to base are spin polarised. As such, the emitter efficiency is dependent on the number of electrons available to flow into the base. Hence, the current amplification $\beta=\Delta \mathrm{I}_{\mathrm{C}} / \Delta \mathrm{I}_{\mathrm{B}}$ can be controlled, not only by the spin polarisation in the base, but also by the nonequilibrium spin in the emitter. This additional dependence of $\beta$ in an MBT is called [9] magnetoamplification. 

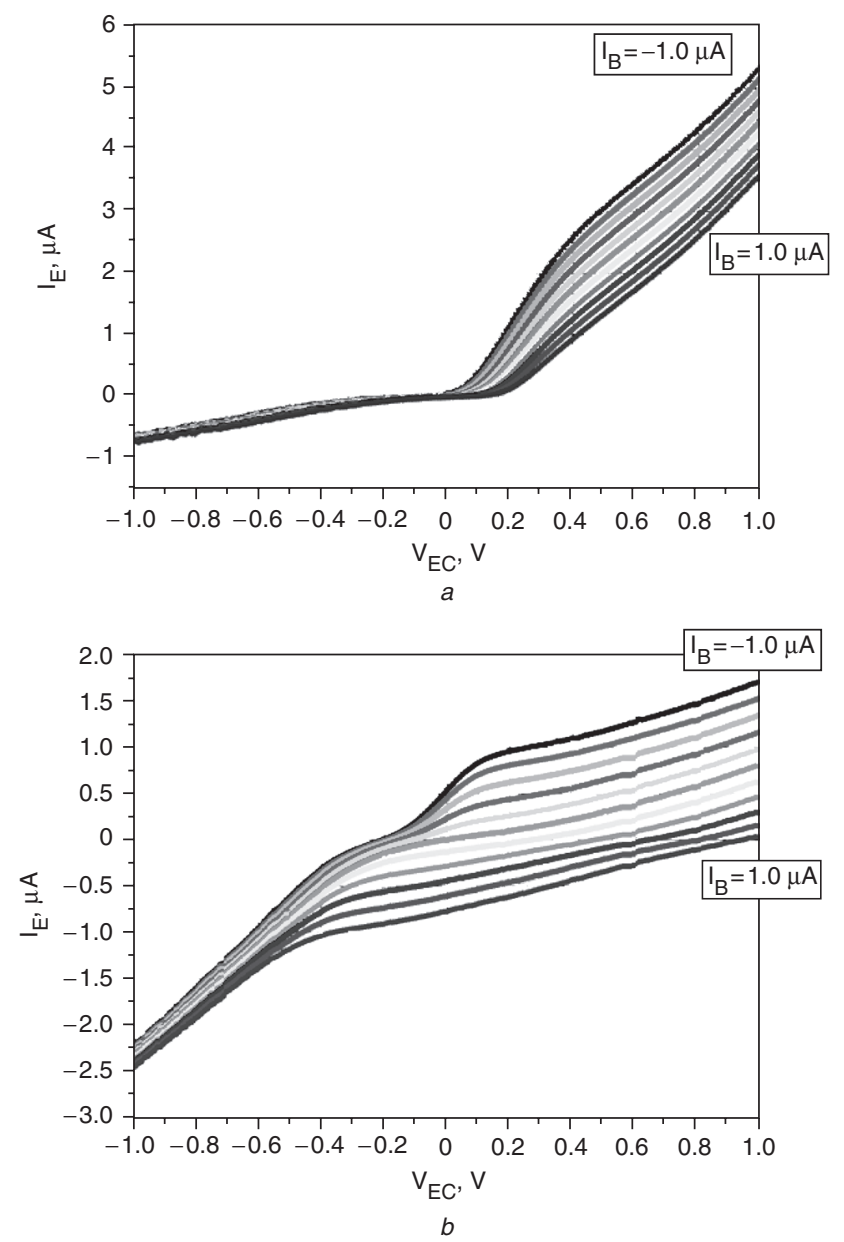

Fig. $12 I_{E}-V_{E C}$ characteristics of spin diffusion transistors in common-collector configuration and zero applied field

Load resistor $R_{L}=9985 \Omega$. The step size of $I_{B}$ is $0.2 \mu \mathrm{A}$ with the starting and ending currents as indicated in the boxes

a Common collector configuration of p-type Southamptom spin transistor (II-6), $\mathrm{H}=0$ Oe, $\mathrm{R}_{\mathrm{L}}=9.985 \mathrm{k} \Omega$

$b$ Common collector configuration of n-type Southamptom spin transistor (II-8), $\mathrm{H}=0 \mathrm{Oe}, \mathrm{R}_{\mathrm{L}}=9.985 \mathrm{k} \Omega$

\section{General operating principles of spin diffusion transistor}

In the preceding Section, we summarised the state of the art in three-terminal spintronics. These devices comprised both all-metal and hybrid metal-semiconductor transistors. The fabricated devices all have a common feature, namely that the spin selectivity/spin transport is limited to the metallic components of the device. In this paper, we discuss a different design, which is distinguished by two main features. First, the semiconductor does more than simply control the distribution of applied voltages; the operation of the device depends on spin transport within the semiconductor itself. Secondly, it is the only spin transistor design capable of current gains equal to or in excess of unity (against other spin transistors which have a current gain of less than $10^{-3}$ ). Reference [13] illustrates why this low current gain is the main sticking point for industrial applications, as well as some of their other limitations. In the remainder of the paper, we discuss the fabrication and characterisation of this new spin diffusion transistor.

The basic operation of this device is similar to a bipolar junction transistor (although it is most closely related to the classical tunnel transistor $[14,15])$. This spin diffusion transistor injects a spin-polarised current from the emitter

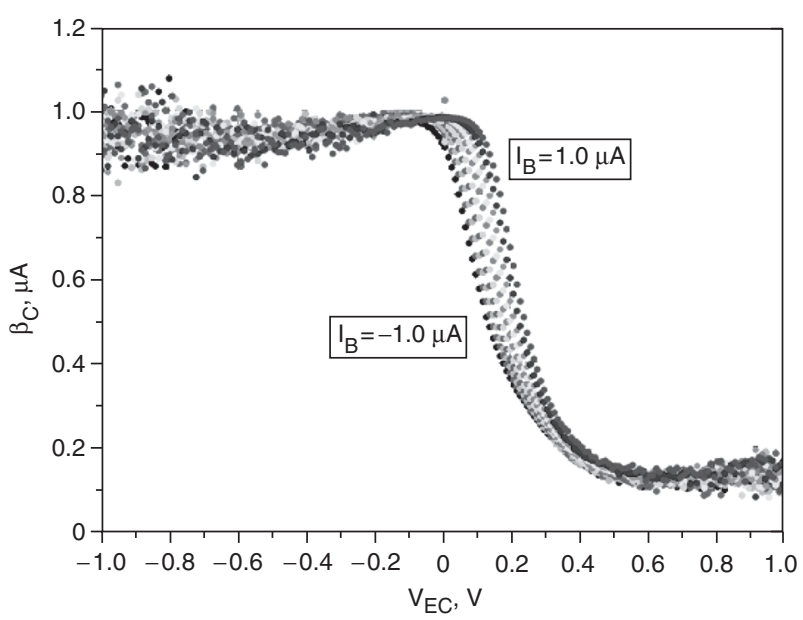

a

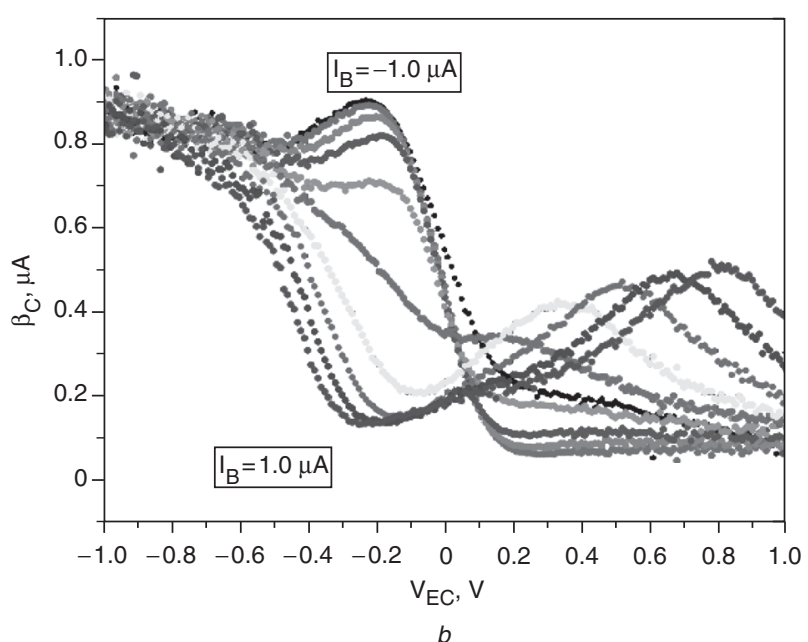

Fig. 13 Calculated current gain at zero field as a function of emitter-collector voltage

For p-type and n-type spin diffusion transistors in zero applied magnetic field. The step size of $\mathrm{I}_{\mathrm{B}}$ is $0.2 \mu \mathrm{A}$ with the starting and ending currents as indicated in the boxes. The current gain is determined by calculating the change in collector current for change in base current and was done between adjacent pairs of base currents (as shown in the legend) at all voltages

$a$ Collector current gain of p-type Southampton spin transistor (II-6) in common collector configuration: $\mathrm{H}=0$ Oe; $\mathrm{R}_{\mathrm{L}}=9.976 \mathrm{k} \Omega$ $b$ Collector current gain of n-type Southampton spin transistor (II-6) in common collector configuration: $\mathrm{H}=0 \mathrm{Oe} ; \mathrm{R}_{\mathrm{L}}=9.976 \mathrm{k} \Omega$

into the electric-field-screened base region. The current which diffuses across the base is driven primarily by a carrier concentration gradient, which forces the carriers injected by the emitter to wander towards the base along the top of an extended energy barrier, at the bottom of which lies the collector. This energy barrier is also spinselective (according to the magnetic orientation of the collector) and determines if these polarised carriers are allowed to fall into the collector or not. Thus, we have a device with a respectable current gain from which power gain may be derived, but whose characteristics may be switched by manipulating the spin selectivity of the energy barrier via an externally applied magnetic field. In this particular device (see Fig. 6), tunnel barriers are used to provide the electric-field shielding of the semiconductor base. However, as outlined in [16], a wide variety of designs are possible in principle, including variants that use $\mathrm{p}-\mathrm{n}$ junctions, Schottky barriers or spin tunnel junctions. 


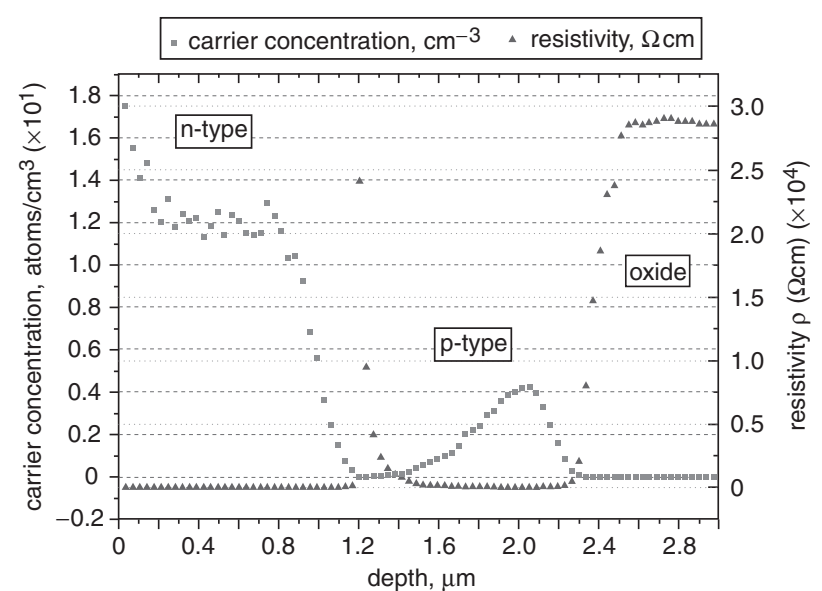

Fig. 14 Spreading resistance data for p-type spin diffusion transistor

These data were reported by Solecon Laboratories Inc. on a sample of the wafer used to make the p-type transistor. Notice that the doping is neither uniform nor of the same type throughout the device. In particular, note that there exists a weak $\mathrm{p}-\mathrm{n}$ junction at a depth of $1.2 \mu \mathrm{m}$

\section{First generation of spin diffusion transistors}

\subsection{Processing}

The samples were fabricated using standard photolithography on n- and p-type silicon-on-insulator (SOI) wafers with a measured resistivity of $2-1000 \Omega-\mathrm{cm}$. The details of the process are specified in $[17,18]$, so only the relevant aspects are provided here. The base contacts were heavily doped to form ohmic contacts. Tunnel barriers of $\mathrm{Si}_{3} \mathrm{~N}_{4}$ were deposited on the collector and emitter contacts by low-pressure epitaxy. Then, all three contacts had $30 \mathrm{~nm}$ of $\mathrm{Co}$ and $1 \mu \mathrm{m}$ of $\mathrm{Al}$ (for the electrical contacts) deposited by sputtering. The resulting structure is shown in Fig. 7.

\subsection{Electrical characteristics in zero applied magnetic field}

This device has been examined in detail in $[17,18]$, the main points are only summarised here for comparison with the second generation.

4.2.1 Two-terminal I-V characteristics: The $\mathrm{I}-\mathrm{V}$ characteristics of the collector-to-emitter (CE) circuit and the collector-to-base $(\mathrm{CB})$ circuit were performed at room temperature in the circuit configurations shown in Figs. $8 a$ and $b$. Typical results are shown in Figs. 9 and 10, and differ slightly between the two types of transistors, although the overall form is the same in both. As shown previously [17, 18], these tunnel barriers conduct at low voltages $(<\sim 0.5 \mathrm{~V})$ by Mott's variable range hopping conduction $[19,20]$, and at higher voltages $(>\sim 0.5 \mathrm{~V})$ by Fowler-Nordheim tunnelling [21]. The existence of two different conduction methods is significant, because it has been well-established experimentally [22] that hopping conduction destroys the spin polarisation of carriers (as the time it takes to hop from one state to the next can exceed the spin lifetime of the carrier). Hence, no magnetic sensitivity should be observed in the hopping conduction regime.

4.2.2 Three-terminal I-V characteristics: When connected in common collector configuration (see Fig. 8c), the transistor exhibits similar characteristics (see Figs. 11, 12) to that of a conventional bipolar

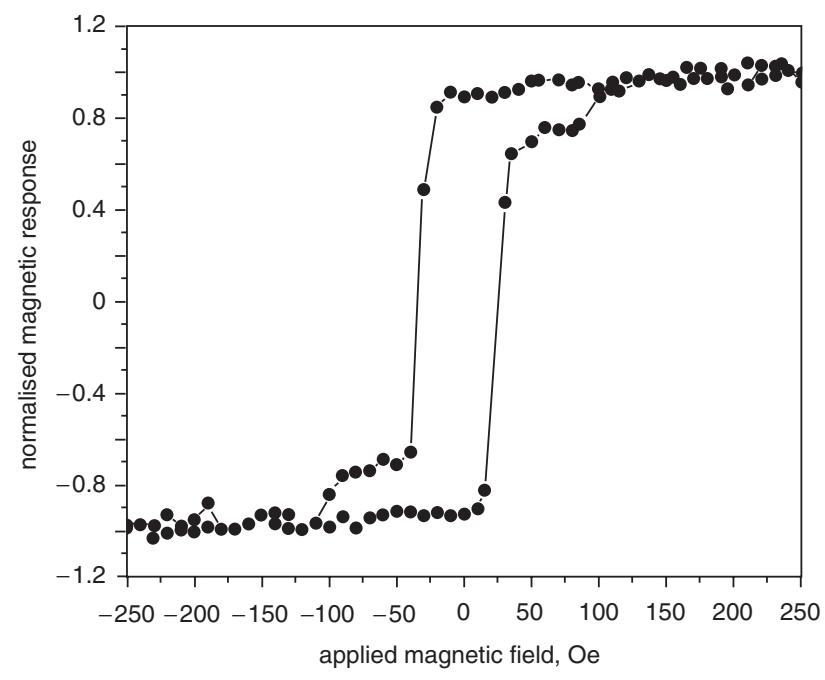

a

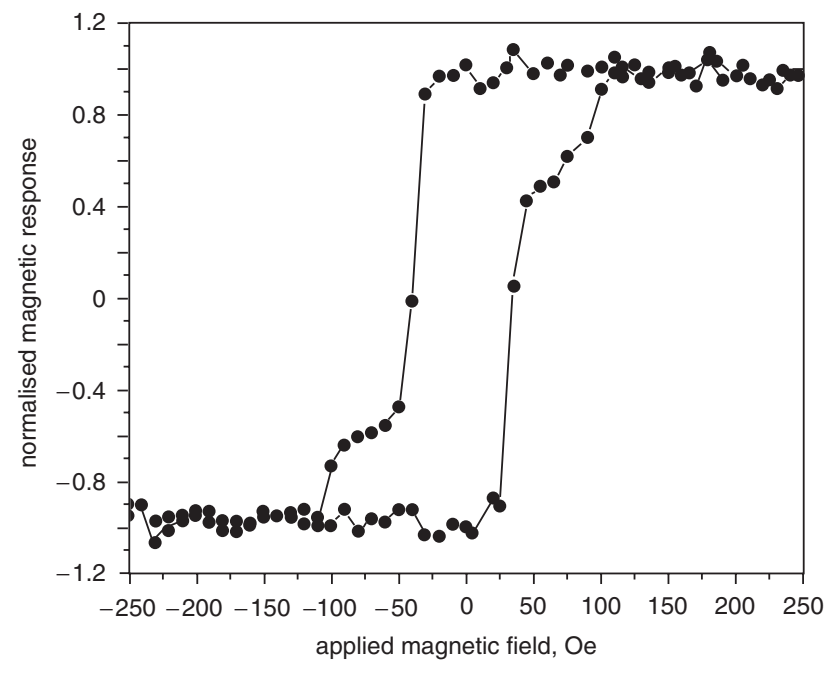

$b$

Fig. 15 Normalised magnetic response minus Si background of spin diffusion transistors

Owing to shape anisotropy, the collector switches at $\sim|30|$ Oe and the emitter and base at $\sim|115|$ Oe. This means that the magnetic moments of the emitter and collector Co layers are parallel for applied magnetic fields from -115 Oe to +30 Oe and greater than $+115 \mathrm{Oe}$, and are antiparallel for positive applied magnetic fields between $30 \mathrm{Oe}$ and $115 \mathrm{Oe}$

$a$ p-type spin diffusion transistor

$b$ n-type spin diffusion transistor

transistor: a dependence on both the base current and the emitter-collector voltage. However, the difference in the $\mathrm{I}-\mathrm{V}$ characteristics between the $\mathrm{n}$ - and $\mathrm{p}$-type transistors can be explained by either different doping in the silicon resulting in different minority carriers traversing the base or electron domination of the tunnelling process (due to the difference in effective masses for electrons and holes), causing one device to be a majority carrier device and the other to be a minority carrier device.

The emitter current as a function of base current and emitter-collector voltage is as high as $-1.56 \mu \mathrm{A}(-3.09 \mu \mathrm{A})$ for p-type (n-type) spin diffusion transistor, which occurs at $V_{\mathrm{EC}}=-1 \mathrm{~V}$ and $I_{\mathrm{B}}=-1.0 \mu \mathrm{A}$. Not only is this a higher output current than in the metal-based devices (by 3 orders of magnitude), but it also occurs at a lower voltage. At a slightly higher base current of $-0.6 \mu \mathrm{A}$ (see Fig. 13), the current gain $(\beta)$ is $1.03 \pm 0.03(0.96 \pm 0.03)$ for $p$-type (n-type). At a base current of $-0.8 \mu \mathrm{A}$, the current gain $(\beta)$ is $1.06 \pm 0.05$ for the p-type transistor. Furthermore, the 

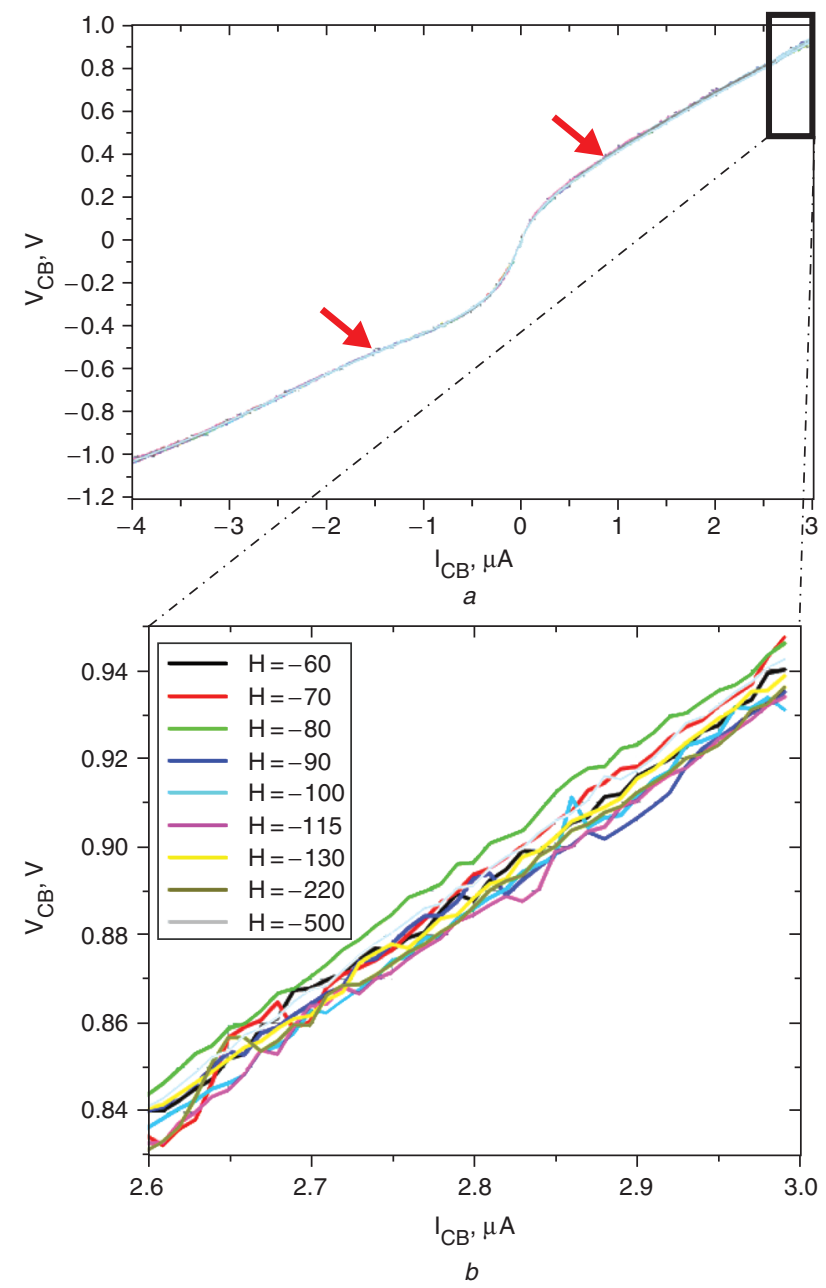

Fig. 16 Two-terminal characteristics of p-type spin diffusion transistor as a function of applied magnetic field from collector to base

Note the 'activity' (oscillations) around the coercive fields. (These samples were saturated at positive fields.)

$a$ Full V-I curve where the arrows show the onset of FowlerNordheim tunnelling

$b$ Blow-up of the highlighted regions in $a$ to show the detail

transfer ratio $\left(\alpha=\mathrm{I}_{\mathrm{E}} / \mathrm{I}_{\mathrm{C}}\right)$ is calculated to be one within error for positive and negative $\mathrm{V}_{\mathrm{EC}}$, for both the p-type and ntype transistor at $I_{\mathrm{B}}=0 \mu \mathrm{A}$. This means that all of the emitter current is being transferred into the collector current. Hence, as the current gain is not identically equal to 1 , the base current must be modifying either the amount of recombination in the $\mathrm{Si}$, or the current injected into the $\mathrm{Si}$ from the emitter.

The transistor action is due solely to the presence of the silicon, because it occurs at any and all applied magnetic fields, and not to any spin transport in the silicon. It depends on a nonequilibrium condition (a surplus of minority carriers) being established at the first tunnel barrier, and continuing to the second barrier. In an ideal bipolar (tunnel) transistor, these minority carriers would diffuse across the base, as almost all of the applied voltage would be dropped over the depletion region of the $p-n$ junctions (the tunnel barriers). However, this device does not have a uniform field in the base, resulting in a position dependence of the base minority carrier density. This is supported by the spreading resistance data (see Fig. 14), which indicate a nonuniform doping profile as well as a weak $\mathrm{p}-\mathrm{n}$ junction in the silicon base. This inhomogeneity manifests itself as a parasitic resistance of $200 \mathrm{k} \Omega(980 \mathrm{k} \Omega)$ for the p-type (n-type) transistor. The presence of this

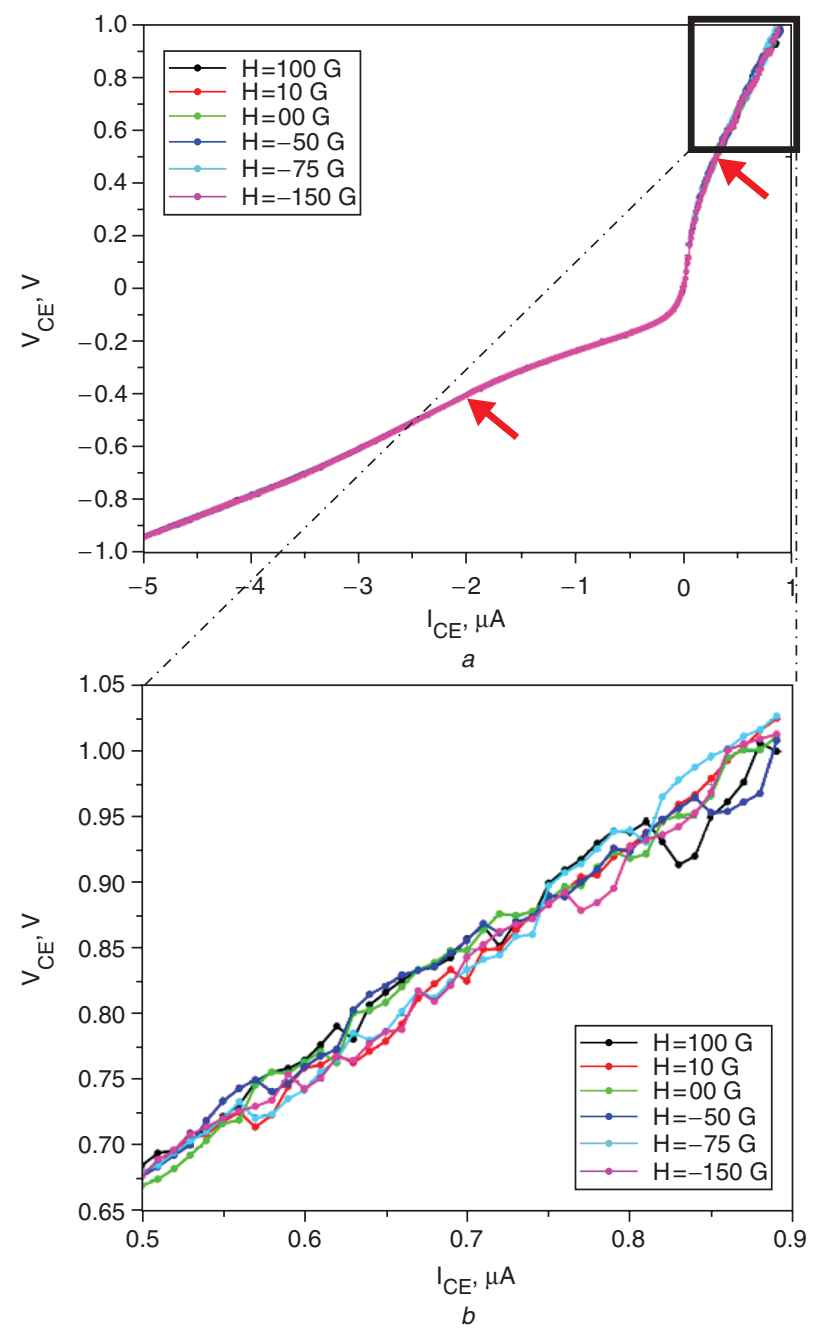

Fig. 17 Two-terminal characteristics of p-type spin diffusion transistor as a function of applied magnetic field from collector to emitter

$a$ Full V-I curve where the red arrows show the onset of FowlerNordheim tunnelling

$b$ Blow-up of the highlighted regions in $a$ to show the detail. Note the 'activity' (oscillations) around the coercive fields. (These samples were saturated at positive fields.)

parasitic base resistance limits the total output current and the current gain; in particular, the tunnel barrier ceases to dominate the output current at $\sim 0.4 \mathrm{~V}(\sim 0 \mathrm{~V})$. This corresponds with the IV characteristics, which are a straight line above $0.4 \mathrm{~V}(\sim 0 \mathrm{~V})$ for the p-type (n-type) device, as well as with the location of the decrease in the current gain.

\subsection{Electrical characteristics in an applied magnetic field}

The magnetic response of the Co layers in the spin diffusion transistors was measured using a vibrating sample magnetometer (VSM). These hysteresis loops (see Fig. 15) indicate that differential switching is occurring in the devices, where the collector contact switches at the lower field of $\sim|30|$ Oe, and the emitter and base switch at the higher field of $\sim|115|$ Oe. Application of a magnetic field is expected to affect the I-V characteristics in two ways. First, the magnetisation of the emitter and collector Co contacts can be differentially manipulated, thereby introducing a spin-selective tunnelling magnetoresistance (TMR) effect that modulates the collector current. Secondly, the applied magnetic field decreases the mean free path in the silicon base via Lorentz magnetoresistance [23] (LMR) thereby 

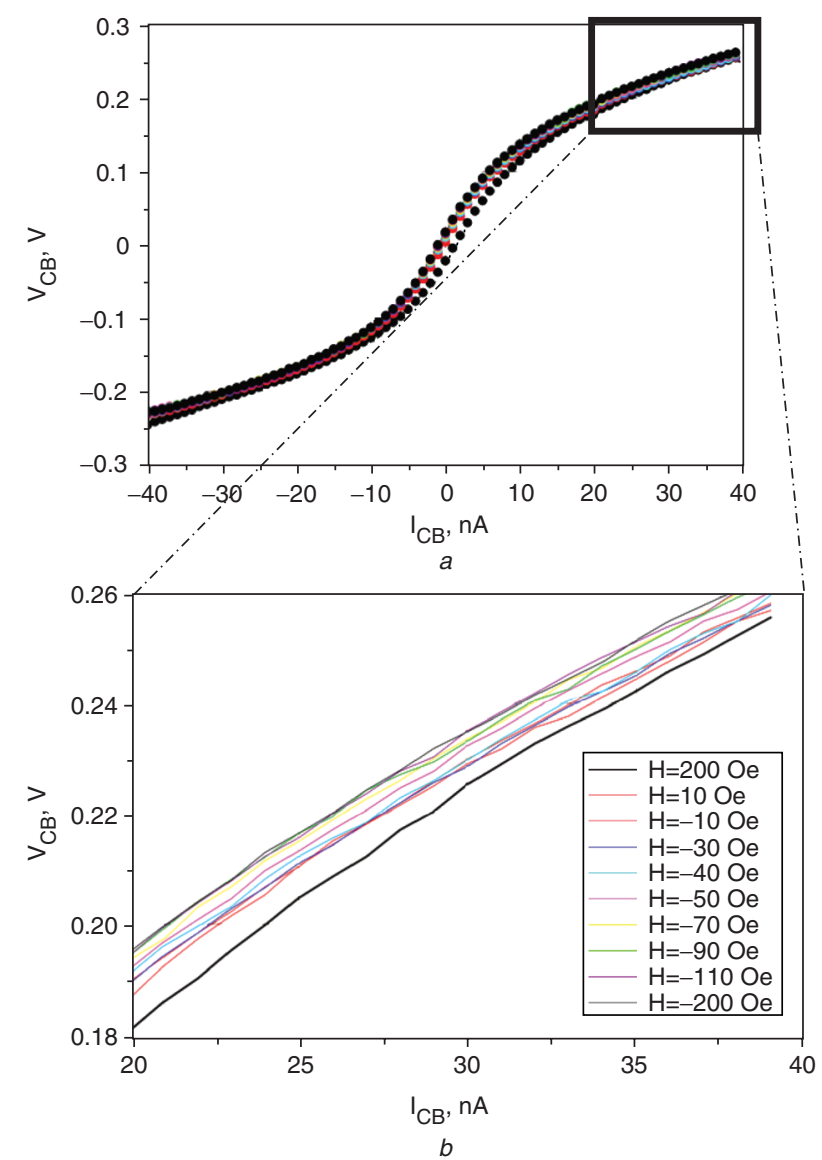

Fig. 18 Two-terminal characteristics of n-type spin diffusion transistor as a function of applied magnetic field from collector to base

Note the 'activity' (oscillations) or lack thereof around the coercive fields. (These samples were saturated at positive fields.)

$a$ Full V-I curve

$b$ Blow-up of the highlighted regions in $a$ to show the detail

also affecting the collector current. Both of these are observed in these devices.

\subsubsection{Two-terminal magnetic I-V character-} istics: The two-terminal measurements of Section 4.2.1 were repeated with a magnetic field applied in the plane of the transistor (perpendicular to the current). There are three important results (see Figs. 16-20) in these measurements. First, the $\mathrm{I}-\mathrm{V}$ characteristics are a function of applied magnetic field. Secondly, no magnetic sensitivity is observed for voltages below the onset of Fowler-Nordheim tunnelling. This concurs with the claim that hopping conduction is occurring at low voltages. Thirdly, most of the 'activity' (shown in the inset of Figs. 16-20 as the ripples or deviations from a straight line) in the electrical characteristics occurs around the magnetic transition region, between -90 and -115 Oe. This suggests that the deviations may be due to magnetic domain formation and/or motion in the Co layers changing the magnetic state seen by different regions of the tunnel barrier.

\subsubsection{Three-terminal magnetic I-V character-} istics: The transistor was again operated in commoncollector mode with the magnetic field applied in the plane of the transistor (perpendicular to the current). The results in Figs. 21-23 are typical and plot the emitter current as a function of applied emitter-collector voltage and magnetic field at $\mathrm{I}_{\mathrm{B}}=-0.6 \mu \mathrm{A}$. These results show a variation in the emitter current as a function of magnetic field, indicating

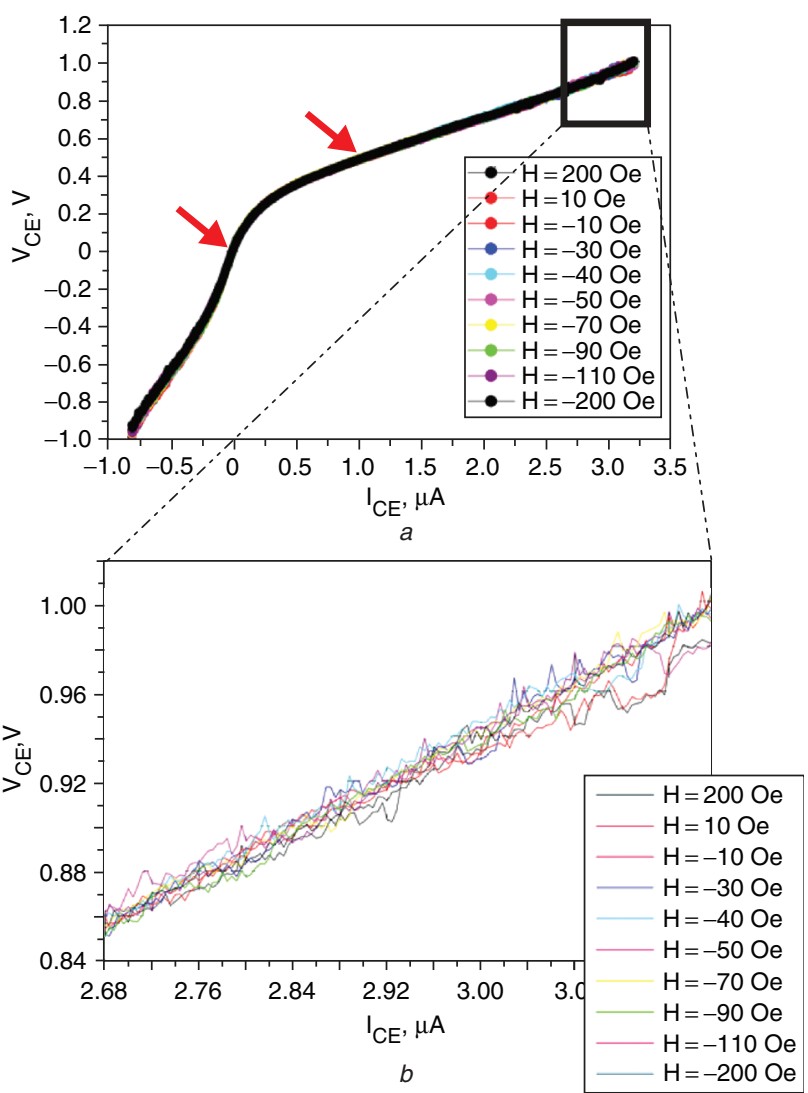

Fig. 19 Two-terminal characteristics of n-type spin diffusion transistor as function of applied magnetic field from collector to emitter

Note the 'activity' (oscillations) or lack thereof around the coercive fields. (These samples were saturated at positive fields.)

a Full V-I curve where the red arrows show the onset of FowlerNordheim tunnelling

$b$ Blow-up of the highlighted regions in $a$ to show the detail

that the transistor behaves as a magnetically tunable device with a field-dependent gain. The maximum variation of the average current gain (where $\beta$ was averaged for all $\mathrm{V}_{\mathrm{EC}}>0.4 \mathrm{~V}$ and for each base current), relative to the current gain at $\mathrm{H}=0 \mathrm{Oe}$, was $-11 \pm 3 \%(-15 \pm 2 \%)$ for $\mathrm{p}$ type (n-type) which occurred at $75 \mathrm{Oe}(110 \mathrm{Oe})$ and $I_{\mathrm{B}}=-0.6 \mu \mathrm{A}$. On examination of the expanded graphs (see Fig. 24) for both n- and p-type at positive $\mathrm{V}_{\mathrm{EC}}$, it is clear that, when the magnetic moments of the emitter and collector Co layers are parallel, the emitter current is larger than in the antiparallel configuration. (From Fig. 21 it would appear that this magnetic sensitivity occurs in the region where the current gain is small. However, the current gain refers to $\mathrm{I}_{\mathrm{C}}$, the collector current and the magnetic sensitivity plotted in Fig. 18 refers to $\mathrm{I}_{\mathrm{E}}$ the emitter current. Due to BJT definitions of positive current, one is turned 'on' in $+\mathrm{V}_{\mathrm{EC}}$ and the other is turned 'on' in $-\mathrm{V}_{\mathrm{EC}}$. Therefore, the region of large current gain is the same as the region of large magnetic sensitivity. The emitter current is plotted to show spin injection since the electrons actually flow from the collector to emitter. The same effect is apparent in the collector current due to conservation of charge.)

A detailed analysis of the origins of the magnetic sensitivity is explained in $[17,23]$, including other possibilities besides spin injection (or TMR) such as LMR, anisotropic magnetoresistance (AMR) and fringing fields from the magnetic elements. LMR is definitely measured, while AMR is eliminated, due to the size of the effect, and 


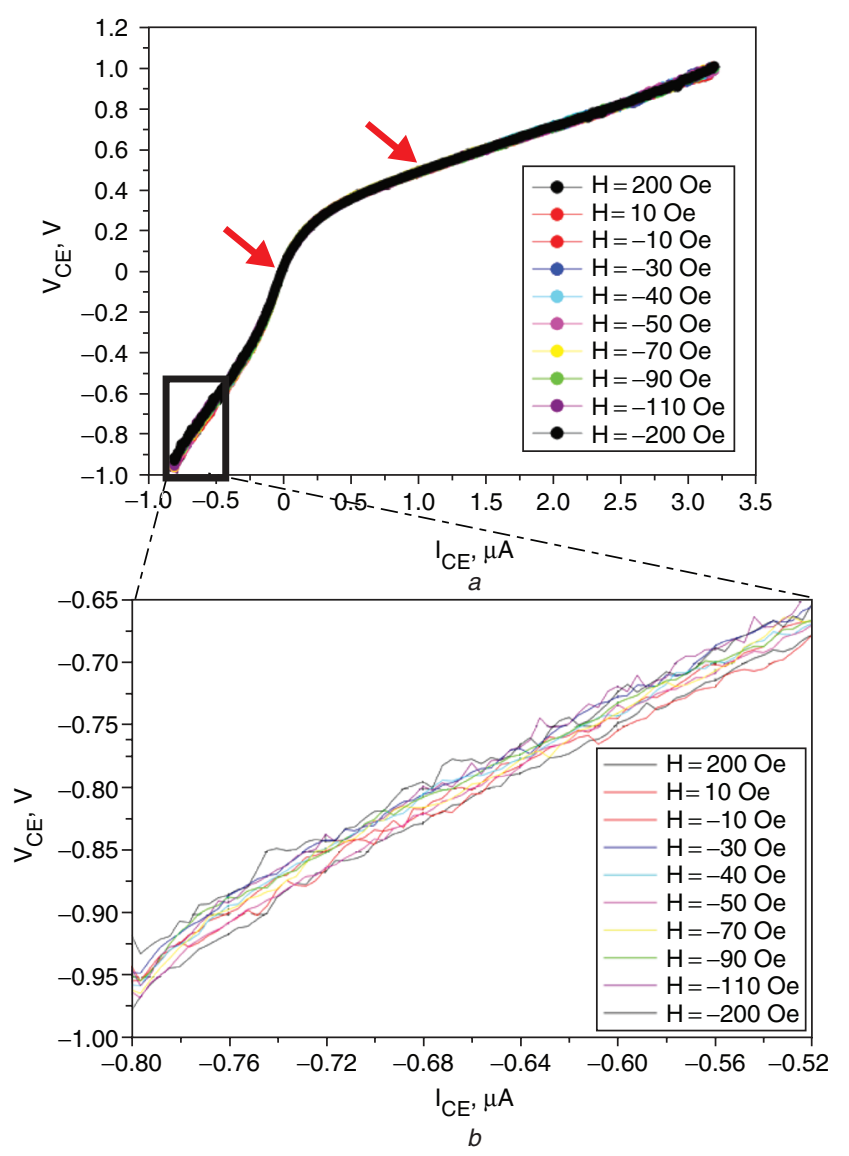

Fig. 20 Two-terminal characteristics of n-type spin diffusion transistor as function of applied magnetic field from collector to emitter

Note the 'activity' (oscillations) or lack thereof around the coercive fields. (These samples were saturated at positive fields.)

$a$ Full V-I curve where the red arrows show the onset of FowlerNordheim tunnelling

$b$ Blow-up of the highlighted regions in $a$ to show the detail

the Hall Effect from fringe fields is eliminated by symmetry considerations. In particular, the percentage change as a function of field from LMR is $4 \%$ of the overall resistance at $|200|$ Oe. This is a change of $\sim 40 \mathrm{k} \Omega$, which originates in part from the parasitic resistance of $200 \mathrm{k} \Omega(980 \mathrm{k} \Omega)$ for the p-type (n-type) device.

Assuming the existence of spin injection into $\mathrm{Si}$ (as presented in [24]), the spin polarisation can be estimated from Julliere's model [25] for spin tunnelling to be $2.5 \pm 0.5 \%$ for the p-type and $10 \pm 1 \%$ for the n-type (see Fig. 25). This value for the spin polarisation is significantly lower than the theoretical value of $38 \%$, as well as being lower than typical values in the literature. This is to be expected as hopping conduction, though no longer dominant, is still active at high tunnel barrier bias and it destroys part of the injected spin polarisation.

\section{Second generation of spin diffusion transistors}

\section{1 Processing}

These samples were identical to the previous generation of devices except in the materials sputtered onto the Si. Tunnelling barriers of $\mathrm{Al}_{2} \mathrm{O}_{3}(1.5 \mathrm{~nm})$ were deposited on the back of the wafer and tunnelling barriers of $\mathrm{Al}_{2} \mathrm{O}_{3}(1.0 \mathrm{~nm})$ were deposited on the front of the wafer by sputtering of $\mathrm{Al}$ followed by plasma oxidation. Co $(3 \mathrm{~nm}) / \mathrm{Fe}(6 \mathrm{~nm}) / \mathrm{Cu}$ $(5 \mathrm{~nm}) / \mathrm{Cr}(3 \mathrm{~nm})$ were deposited on the emitter and base contacts, while $\mathrm{CoFe}(3-6 \mathrm{~nm}) / \mathrm{Cu}(5 \mathrm{~nm}) / \mathrm{Cr}(3 \mathrm{~nm})$ were
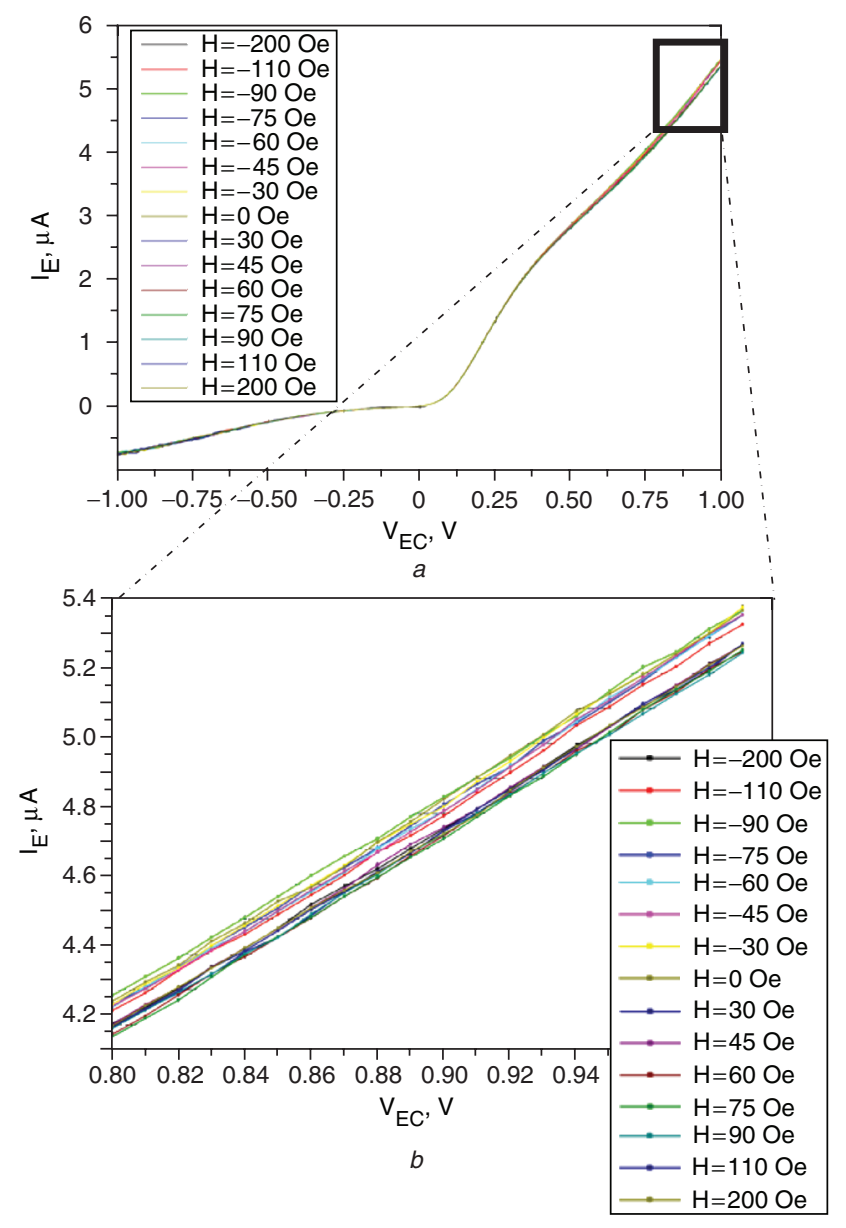

Fig. $21 I_{E}$ characteristics as function of applied magnetic field of p-type spin diffusion transistors in common-collector configuration with $I_{B}=-0.6 \mu \mathrm{A}$

The samples were saturated at negative fields

$a$ Full V-I characteristics

$b$ Blow-up of the black boxes from $a$ for detail

deposited on the collector. The resulting structure is shown in Fig. 26 and an actual device is shown in Fig. 27.

\subsection{Electrical characteristics in zero applied field}

5.2.1 Two-terminal I-V characteristics: I-V characteristics of the collector-to-emitter (CE) circuit and the collector-to-base (CB) circuit were performed at room temperature in the circuit configurations shown in Fig. $8 a$ and $8 b$. Typical results on the stable barriers are shown in Fig. 28. However, although these VI characteristics show diode-like behaviour at low bias, they are not accurately represented by the equations for either an ideal diode or a nonideal diode as shown in Fig. 28. Instead, away from zero, the electrical characteristics of the tunnel barriers are linear in voltage and current, and are characterised by a resistance of $300 \mathrm{k} \Omega$. This resistance is probably also due to the nonuniformity in the $\mathrm{Si}$ (as discussed in Section 4.2.2), with additional contributions from diffusion of the oxygen or $\mathrm{Al}$ into the surrounding $\mathrm{Si}$, or $\mathrm{Co}$ or $\mathrm{Fe}$ into the $\mathrm{Al}_{2} \mathrm{O}_{3}$.

5.2.2 Three-terminal I-V characteristics: As seen in Fig. 29, these results closely resemble the results of the first generation p-type spin diffusion transistors for positive $\mathrm{V}_{\mathrm{EC}}$. However, there is negligible conduction in the negative $\mathrm{V}_{\mathrm{EC}}$ regime, which results in a collector current 


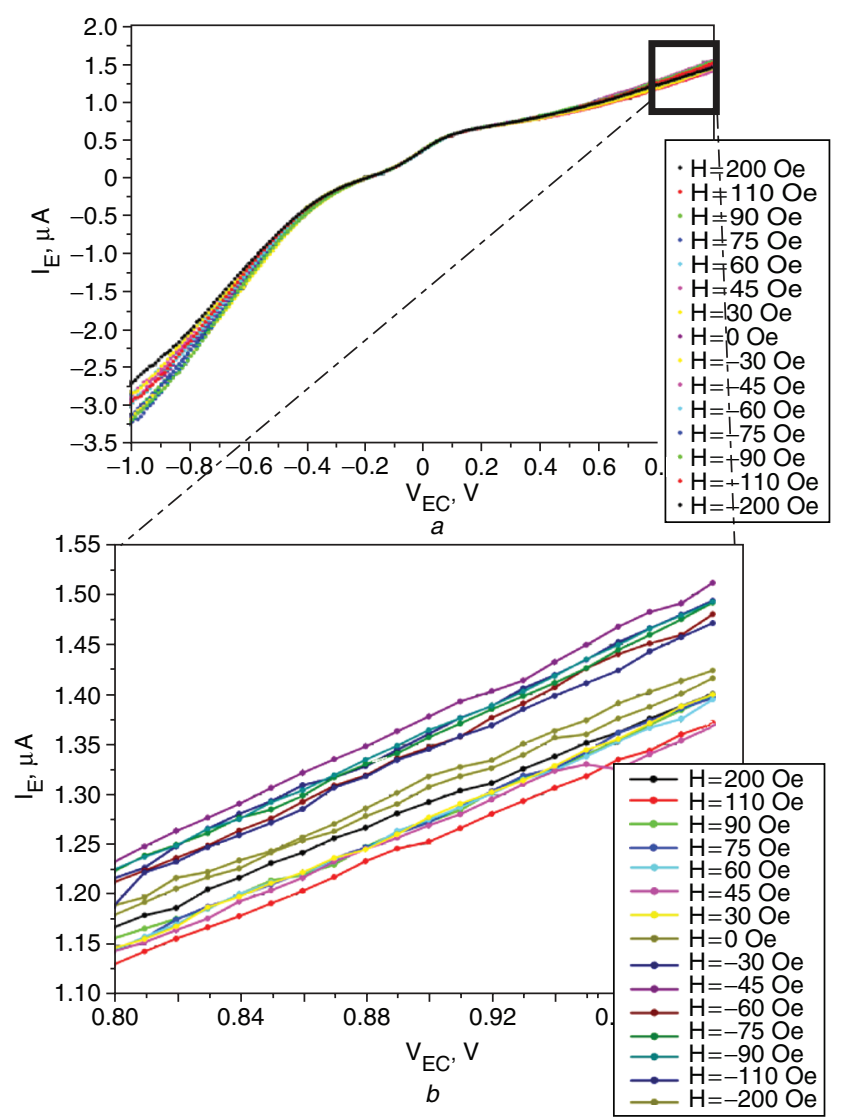

Fig. $22 I_{E}$ characteristics as function of applied magnetic field of n-type spin diffusion transistors in common-collector configuration with $I_{B}=-0.6 \mu \mathrm{A}$

The samples were saturated at negative fields

$a$ Full V-I characteristics

$b$ Blow-up of the black boxes from $a$ for detail

gain for $\mathrm{V}_{\mathrm{EC}}<0 \mathrm{~V}$, identical to 1 . Hence, in this range, the base current does not modify the emitter current (which is zero), it simply adds to it. (For $\mathrm{V}_{\mathrm{EC}}>1 \mathrm{~V}$, the current gain plateaued at an average of $-0.933 \pm 0.010$. This plateau occurs when the contact resistance begins to exceed the tunnel barrier resistance at a voltage of $\sim 0.9 \mathrm{~V}$.)

\subsection{Electrical characteristics in an applied field}

The application of a magnetic field is expected to affect the $\mathrm{I}-\mathrm{V}$ characteristics in the same ways as in the first generation of spin diffusion transistors. However, the primary expected differences are (i) that the cleaner switching will yield clearer parallel and antiparallel conditions and more stable electrical characteristics; (ii) that the larger separation between the coercive fields will yield more distinct parallel and antiparallel conditions and therefore larger TMR values; and (iii) that the new tunnel barriers will conduct spin better leading to a more highly spinpolarised current in the base which will yield greater magnetic sensitivity in the emitter current and larger TMR values.

The magnetic response, as measured by a SQUID Magnetometer, shows three coercivities (see Fig. 30). The base contact switches at $\mathrm{H}_{\mathrm{C}} \sim 10 \mathrm{Oe}$, the emitter contact switches at $\mathrm{H}_{\mathrm{C}} \sim 85 \mathrm{Oe}$, and the collector contact at fields $>100$ Oe.

5.3.1 Two-terminal magnetic I-V characteristics: The two-terminal measurements of Section 5.2.1 were repeated with a magnetic field applied in the plane of

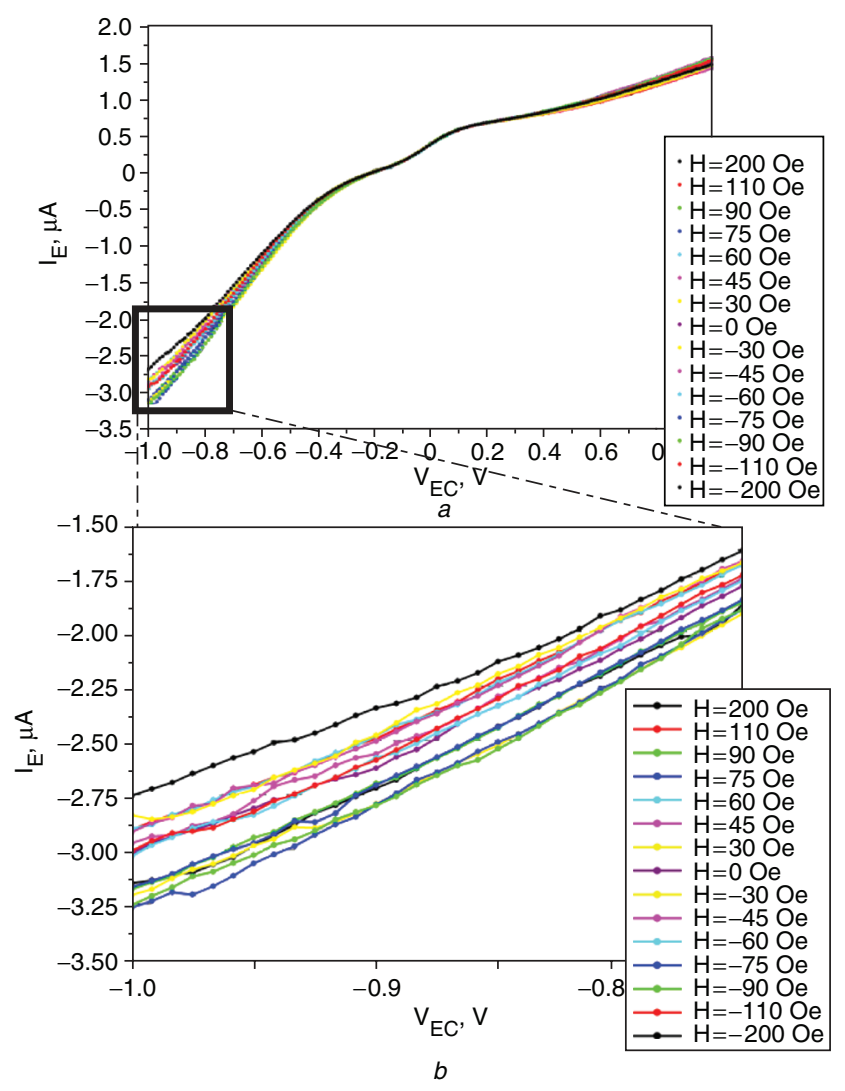

Fig. $23 I_{E}$ characteristics as function of applied magnetic field of n-type spin diffusion transistors in common-collector configuration with $I_{B}=-0.6 \mu \mathrm{A}$

The samples were saturated at negative fields

$a$ Full V-I characteristics

$b$ Blow-up of the black boxes from $a$ for detail

the transistor (perpendicular to the current). There are three important results (shown in Figs. 31 and 32) from these measurements. First, the I-V characteristics are again a function of applied magnetic field. Secondly, as compared to the two terminal magnetic $\mathrm{I}-\mathrm{V}$ characteristics of the first generation of devices, the $\mathrm{I}-\mathrm{V}$ characteristics are much smoother, indicating cleaner magnetic switching than before. Thirdly, the variation in voltage at different applied magnetic fields is as large as $0.0934 \mathrm{~V}$, for the collector to base measurement, and $0.0667 \mathrm{~V}$, for the collector to emitter measurement, which is well outside the error in the measurement of $\pm 0.0001 \mathrm{~V}( \pm 0.1 \mathrm{mV})$.

\subsubsection{Three-terminal magnetic I-V character-}

istics: The transistor was again operated in commoncollector mode with the magnetic field applied in the plane of the transistor (perpendicular to the current). The results (see Figs. 33 and 34) show a variation in the emitter current (of up to $0.672 \pm 0.006 \mu \mathrm{A}$ in $|90| \mathrm{Oe}$ ) as a function of magnetic field, indicating that the transistor behaves as a magnetically tunable device with a field-dependent emitter gain. The maximum relative variation of the emitter current was $-14 \pm 0.3 \%$ which occurred at $-90 \mathrm{Oe}, \mathrm{V}_{\mathrm{EC}}=1 \mathrm{~V}$ and $\mathrm{I}_{\mathrm{B}}=0.0 \mu \mathrm{A}$. Overall, the current variation shows a negative change; the emitter current is being decreased as a function of field.

Close examination of Fig. 34 shows that, although the emitter current for positive $V_{E C}$ varies in an identical fashion to that expected for TMR-influenced data, there is a field-dependent distribution. Hence, the data do not show a simple TMR/spin injection signature (see Fig. 35): one current for parallel contacts and another for antiparallel 


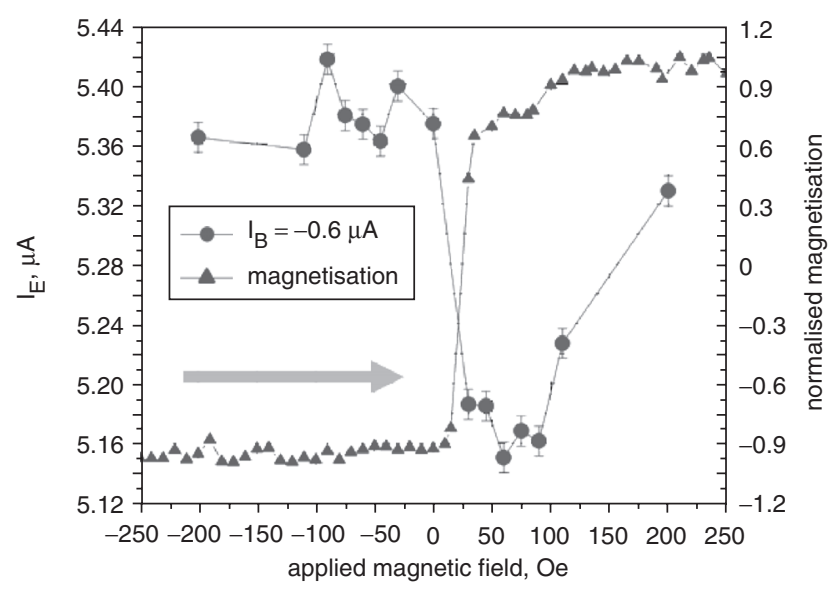

a

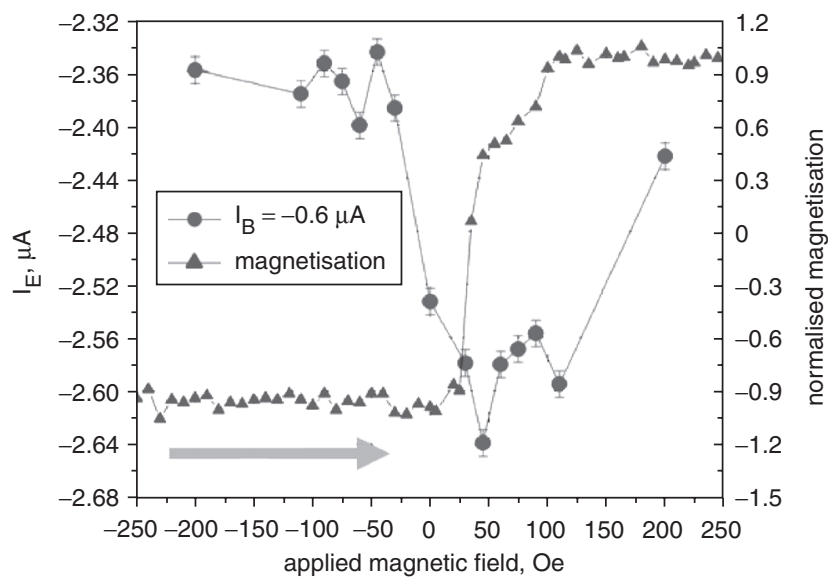

$b$

Fig. 24 Emitter current as a function of applied magnetic field after correction for $L M R$ at $V_{E C}=1 \mathrm{~V}$ and $I_{B}=-0.6 \mu \mathrm{A}$

Half of a hysteresis loop as measured on a VSM is shown by the circular symbols. The arrow indicates the direction of the magnetic field sweep of the measurements, following saturation at fields $<-1$ kOe. (These data points were simply taken from the common-collector configuration data in the previous graph above the HC/FNT threshold, corrected for Lorentz magnetoresistance, and plotted separately for clarity. Owing to the substantial additional noise associated with magnetic field sweeping (due to magnetocaloric effects combined with the temperature dependence of silicon), the data were measured by sweeping the voltage/current characteristics at a selection of fixed magnetic fields.)

a Emitter current (corrected for LMR) as a function of applied magnetic field through p-type $\mathrm{Si}\left(\mathrm{V}_{\mathrm{EC}}=1 \mathrm{~V}\right)$

$b$ Emitter current (corrected for LMR) as a function of applied magnetic field through n-type $\mathrm{Si}\left(\mathrm{V}_{\mathrm{EC}}=1 \mathrm{~V}\right)$

contacts. The two groups have a symmetric contribution, which could be the result of any one of the symmetric contributions discussed in Section 4. Finally, it should be noted that, in both cases, the antiparallel fields yield a larger current than the parallel fields. This is due to Co and CoFe having a different majority spin carrier [26].

By removing these symmetric contributions, the maximum TMR is calculated (see Fig. 36 ) to be $13.3 \pm 0.2 \%$ at $\mathrm{I}_{\mathrm{B}}=-0.2 \mu \mathrm{A}$. Close examination of the TMR shows their dependence on the magnetic response of the transistors. At $|30|$ Oe, the base contact has switched. This introduces some spin-polarised current into the $\mathrm{Si}$. (Recall that the base junction here is a tunnel junction and that the base current is predominantly additive, not recombinative.) Although, the emitter contact does not really start to switch until $75 \mathrm{Oe}$, the magnetisation is dropping slightly. This could lead to the decrease in TMR through 45, 60 and 75, as the
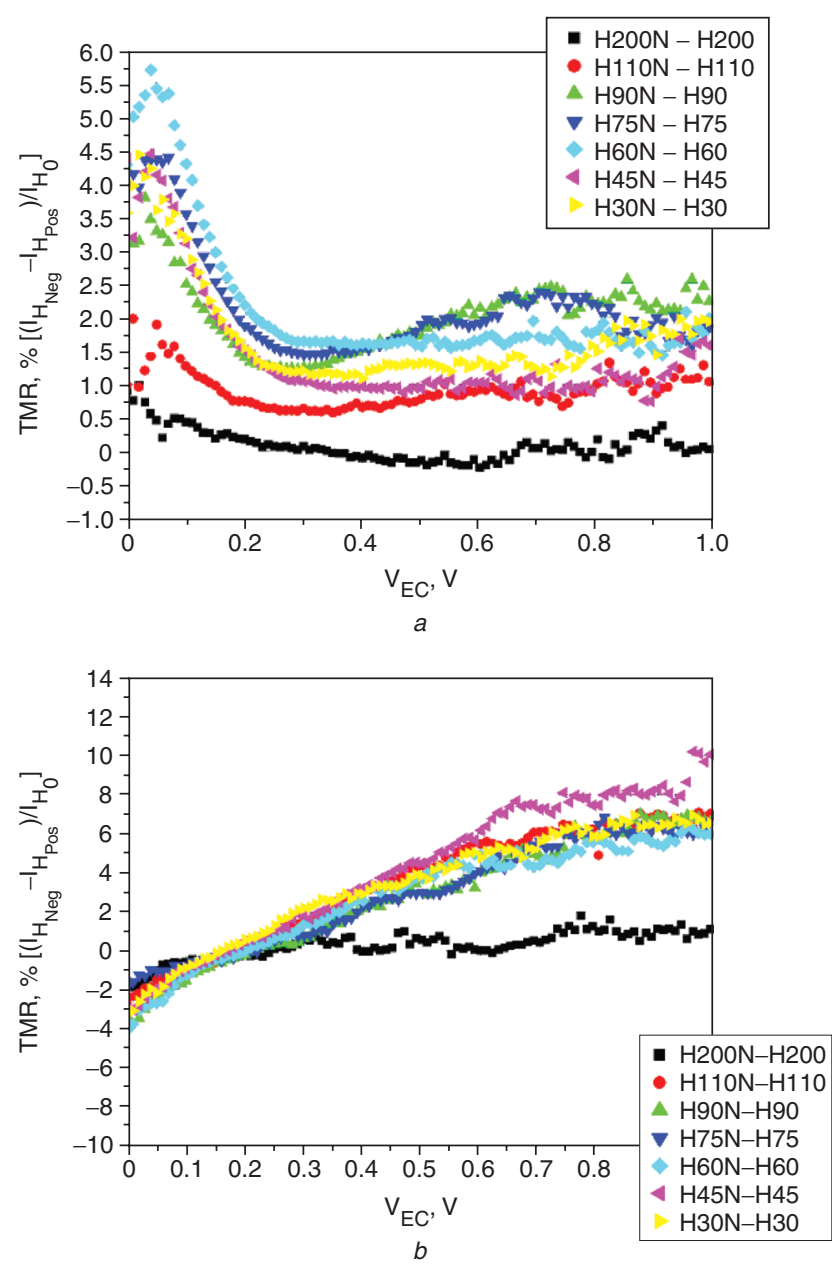

Fig. 25 Calculated TMR of the emitter current for p-type and ntype spin diffusion transistor in common collector configuration and as function of both emitter-collector voltage and applied magnetic field at $I_{B}=-0.6 \mu \mathrm{A}$

The effect is zero for the \pm 200 Oe data since the magnetic elements are in parallel and the noise around $\mathrm{V}_{\mathrm{EC}}=0 \mathrm{~V}$ is the result of division by $\mathrm{I}_{\mathrm{C}} \approx 0 \mathrm{~A}$. The TMR is the difference between the positive and negative field signals, divided by the signal at zero field, and represents only the magnetic sensitivity from spin transport, not from LMR

$a$ TMR percentage for p-type spin transistor (II-6), $\mathrm{I}_{\mathrm{B}}=-0.6 \mu \mathrm{A}$

$b$ TMR percentage for n-type spin transistor (II-8), $\mathrm{I}_{\mathrm{B}}=-0.6 \mu \mathrm{A}$
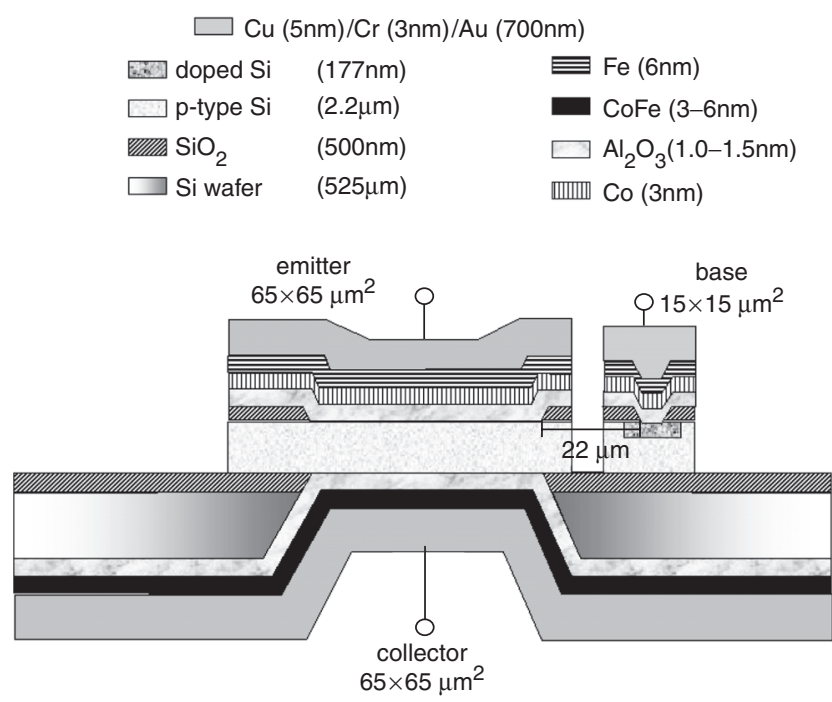

Fig. 26 Schematic diagram of silicon-based spin diffusion transistor

This geometry is identical to that of the first generation of spin diffusion transistors 


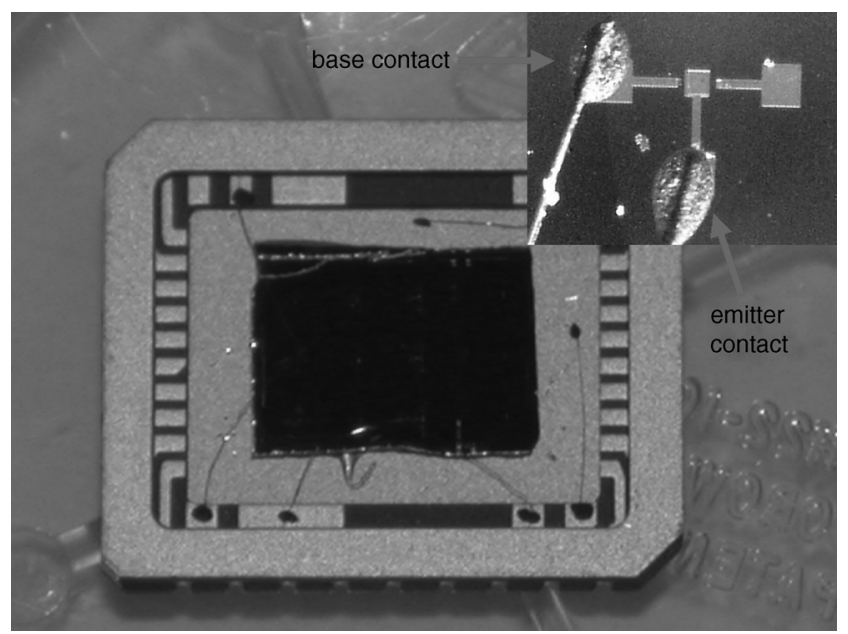

Fig. 27 Actual spin diffusion transistor as mounted in chip package

Inset: Close-up of carbon paste bonding where red arrows indicate the particular contact

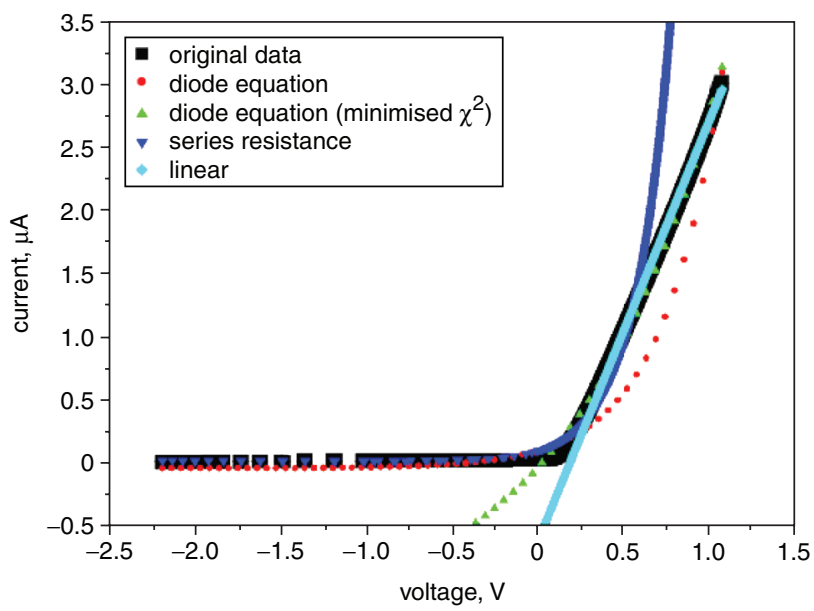

a

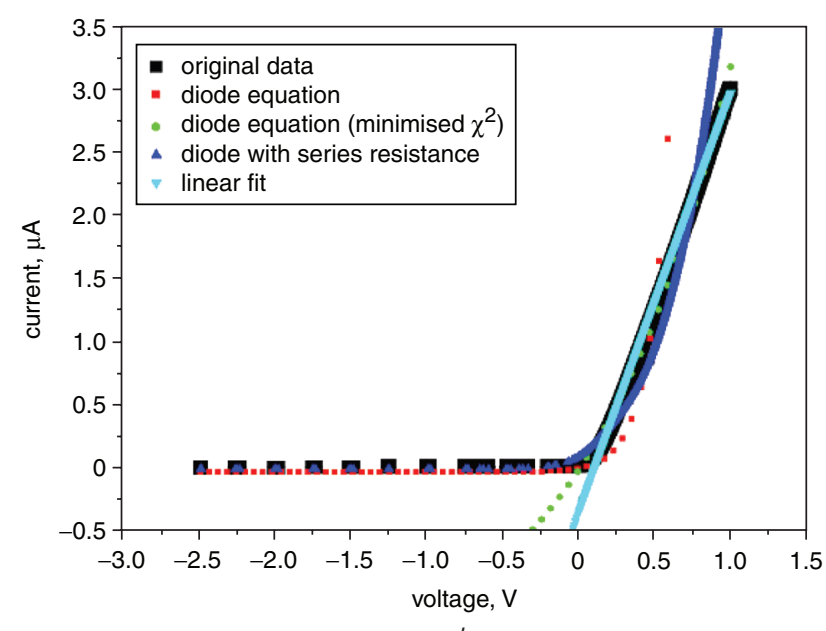

Fig. 28 Two-terminal characteristics of the spin diffusion transistor

The black dots indicate measured data points; the red and green dots are fits to the diode equation; the dark blue dots are fits to diode equation plus a series resistance; and the light blue dots are the linear fits

$a$ V-I curves for Strasbourg transistor III-33: collector to base $b \mathrm{~V}-\mathrm{I}$ curves for Strasbourg transistor III-33: collector to emitter
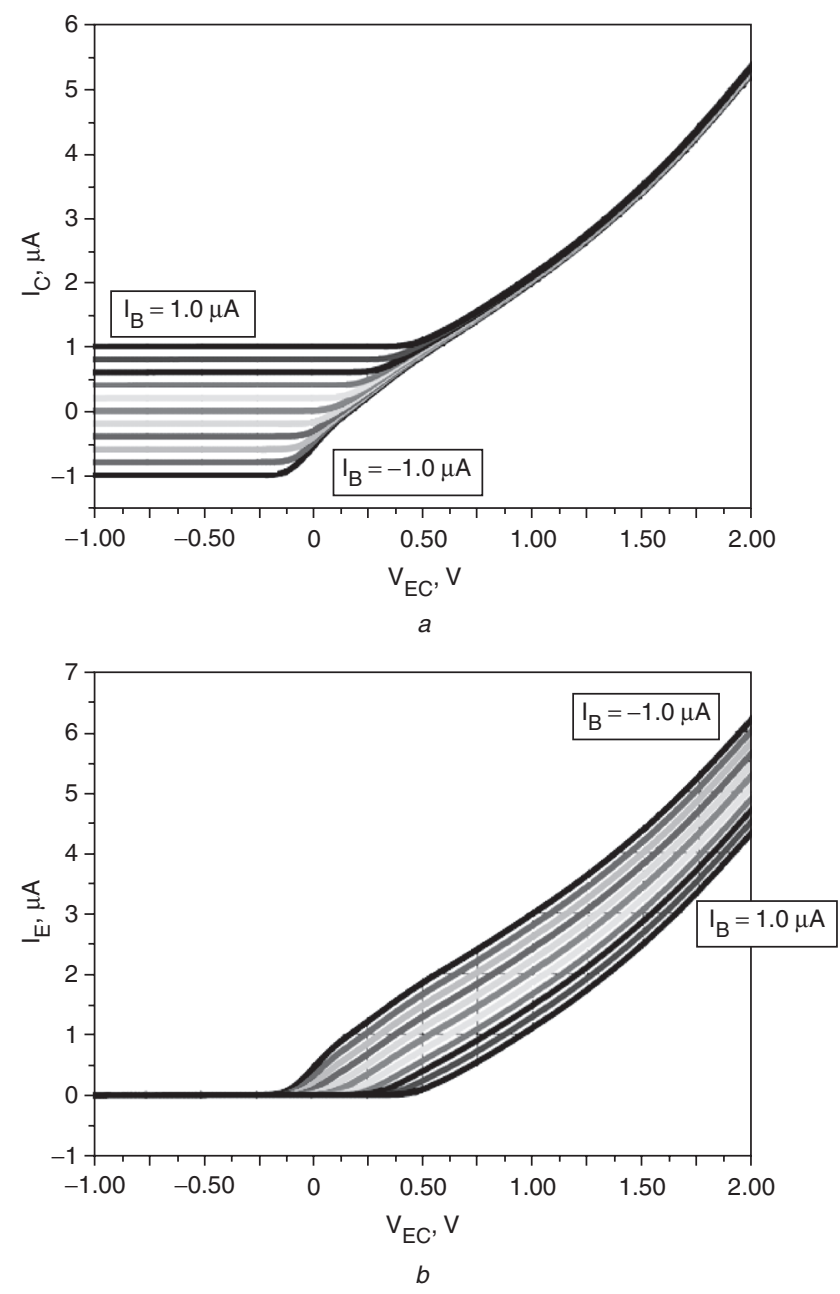

Fig. $29 V-I$ characteristics of the p-type spin diffusion transistor in common-collector configuration and zero applied field

The load resistor $R_{L}=973 \Omega$. The step size of $I_{B}$ is $0.2 \mu \mathrm{A}$ with the starting and ending currents as indicated in the boxes

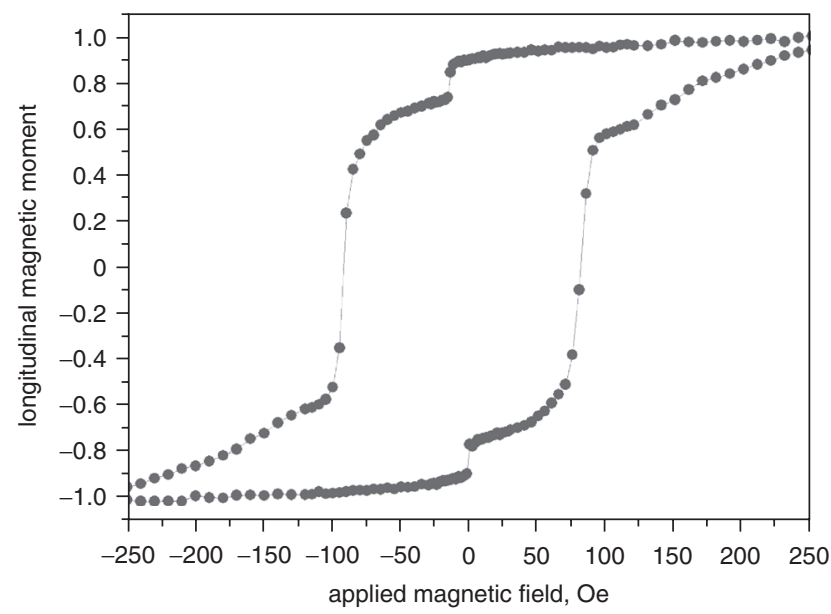

Fig. 30 Magnetic characterisation with three coercivities at $H_{C} \sim 10 \mathrm{Oe}, H_{C} \sim 85 \mathrm{Oe}$ and $\mathrm{H}_{\mathrm{C}}>100 \mathrm{Oe}$

These are hypothesised to belong to the base, emitter and collector contacts, respectively. Magnetic data for Strasbourg transistor III-3 after NRL processing

emitter and base return to parallel alignment. By about 90 Oe, the emitter has almost completely switched, leading to antiparallel alignment of the emitter and collector, and the sudden jump in TMR. For the remaining fields, the collector undergoes a long reversal process, thereby 


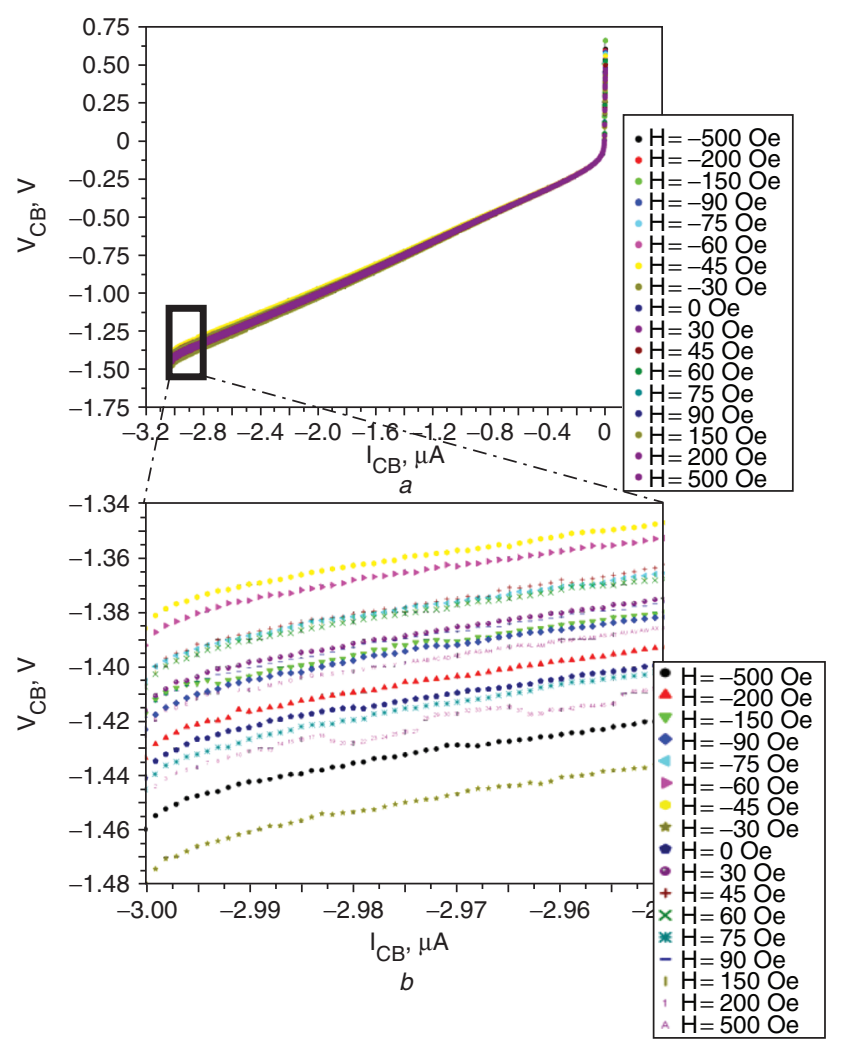

Fig. 31 Two-terminal characteristics in an applied magnetic field of p-type spin diffusion transistor from collector to base These samples were saturated at positive fields $a$ Full $\mathrm{I}-\mathrm{V}$ characteristics

$b$ Blow-up of the highlighted regions in $a$ to show the detail

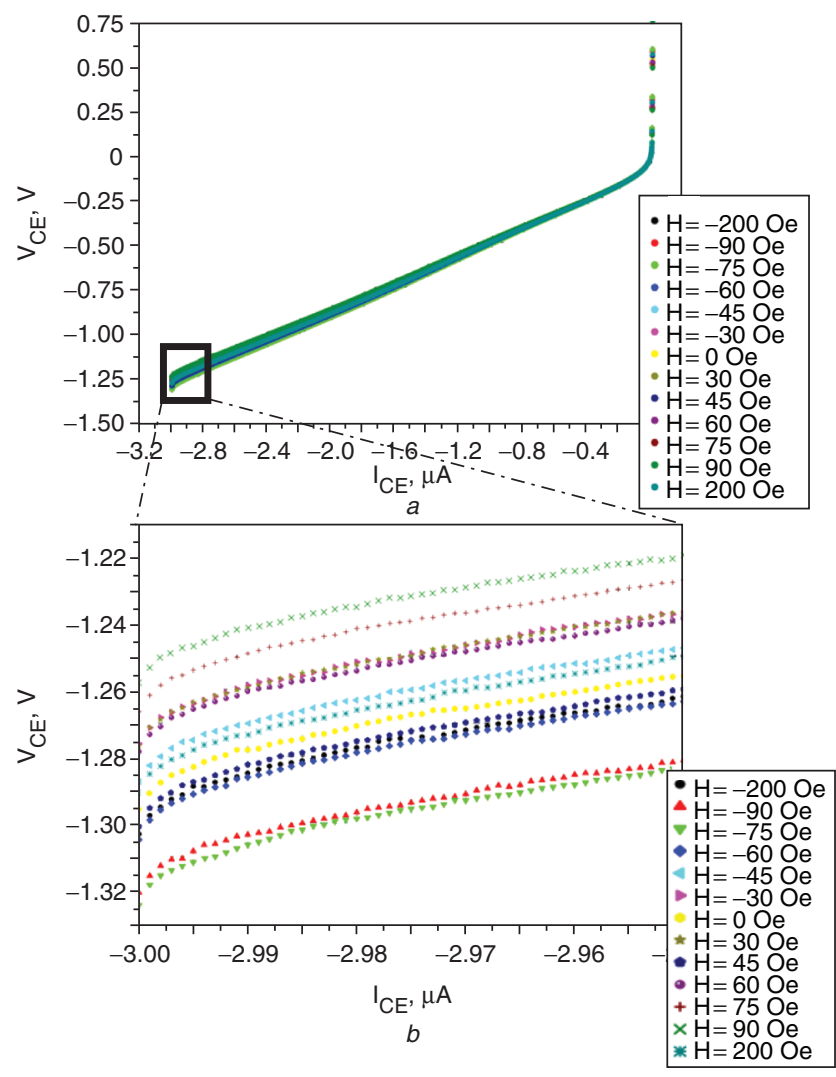

Fig. 32 Two-terminal characteristics in applied magnetic field of p-type spin diffusion transistor from collector to emitter

These samples were saturated at positive fields

$a$ Full $\mathrm{I}-\mathrm{V}$ characteristics

$b$ Blow-up of the highlighted regions in $a$ to show the detail

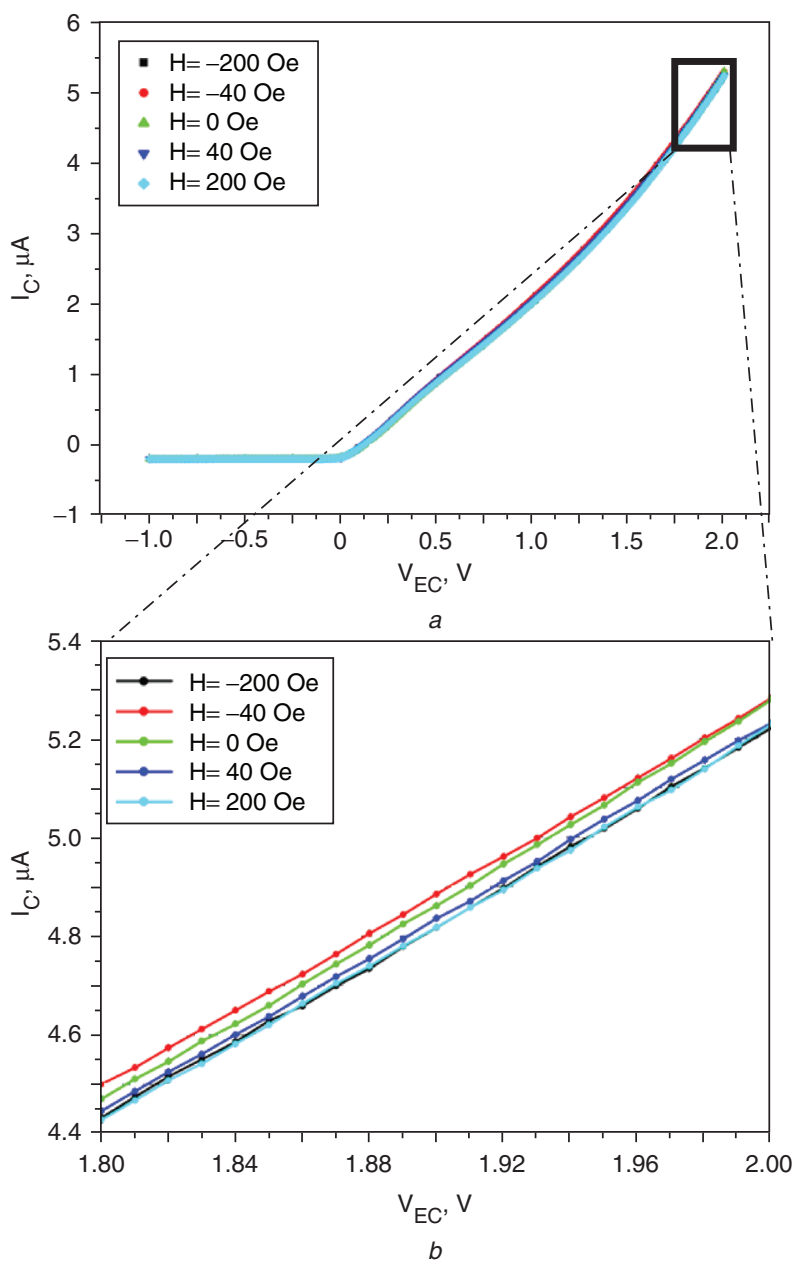

Fig. $33 I_{C}$ characteristics as function of applied magnetic field of spin diffusion transistors in common-collector configuration with $I_{B}=-0.2 \mu \mathrm{A}$

These samples were saturated at positive fields

$a$ Full V-I characteristics

$b$ Blow-up of the square regions in order to show the detail

decreasing the TMR effect. Hence, the TMR is nonzero for $|150|$ Oe and $|200|$ Oe, although the magnitude decreases with the increase in field. The effect is very close to zero for the \pm 500 Oe data because the magnetic elements are nearly all in parallel, as seen from the magnetisation curve shown Fig. 30.

\section{Conclusions}

Proof of concept has been established of a 'high' current gain (greater than unity), magnetically sensitive, silicon-base spin diffusion transistor. (The use of the word 'high' is with respect to other spin transistors.) This device has a current gain slightly greater than unity, which is 3 orders of magnitude larger than any other spin transistor, although it is still less than the typical bipolar junction transistor current gain of 100. However, as was shown in [14], it is possible for this design to achieve current gains comparable to the bipolar junction transistor. In zero magnetic field and at room temperature, the emitter I-V characteristics are similar to those of conventional transistors, and afford a current gain greater than unity and respectable emitter currents. The current gain of the device can be magnetically tuned (up to $-12 \pm 4 \%(-14 \pm 3 \%)$ for p-type (n-type) which occurred at -60 Oe and $\left.\mathrm{I}_{\mathrm{B}}=-0.6 \mu \mathrm{A}\right)$. Moreover, the base current and emitter-collector voltage control this 


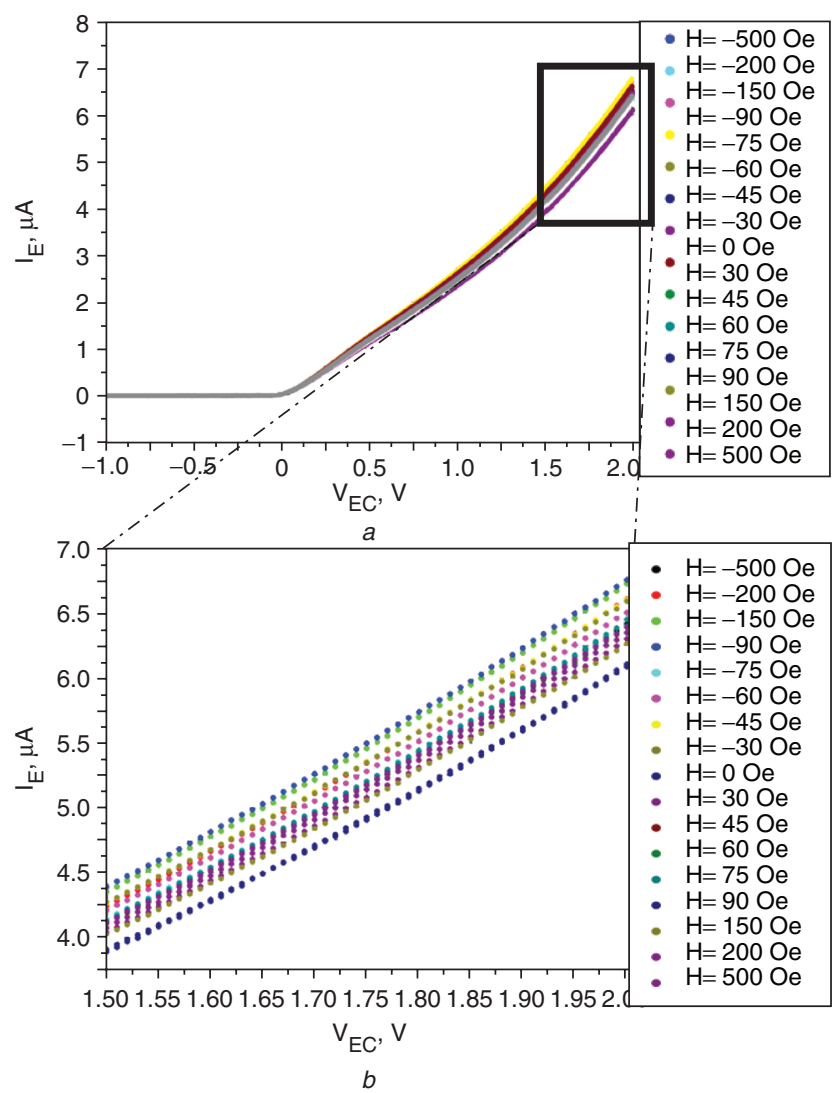

Fig. $34 I_{E}$ characteristics as a function of applied magnetic field of spin diffusion transistors in common-collector configuration with $I_{B}=-0.2 \mu \mathrm{A}$

These samples were saturated at positive fields

$a$ Full V-I characteristics

$b$ Blow-up of the square regions in order to show the detail

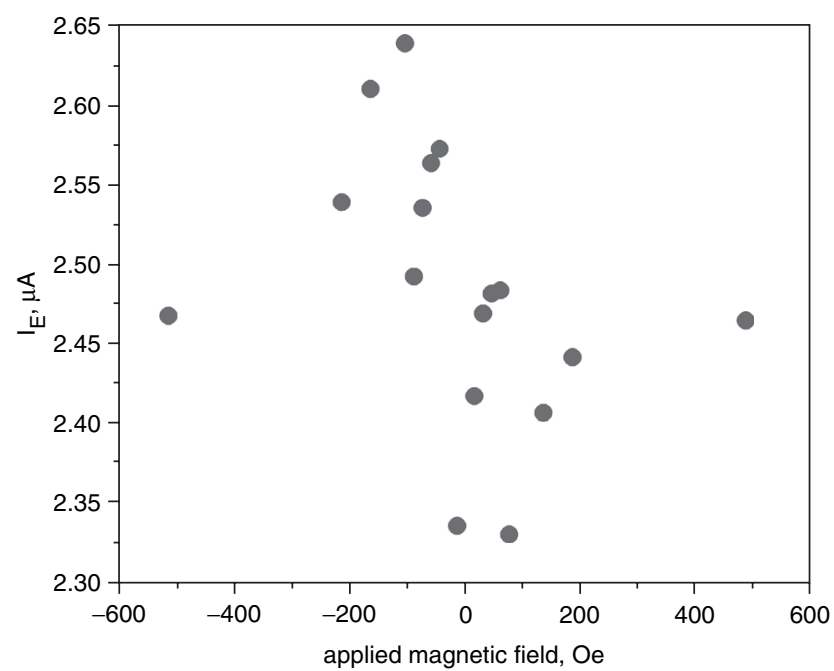

Fig. 35 Emitter current as function of applied magnetic field in common-collector configuration with $I_{B}=-0.2 \mu \mathrm{A}$ and $V_{E C}=1 \mathrm{~V}$ for p-type spin diffusion transistor

These data points were simply taken from the common-collector configuration data in the previous graph and plotted separately for clarity. (The samples were saturated at positive fields.)

field-dependent gain. However, the device described has a number of shortcomings, which, if eliminated, may provide improved performance. These include:

- Nonoptimal tunnel barriers: $\mathrm{Si}_{3} \mathrm{~N}_{4}$ tunnel barriers conduct initially via hopping conduction, which is well known to

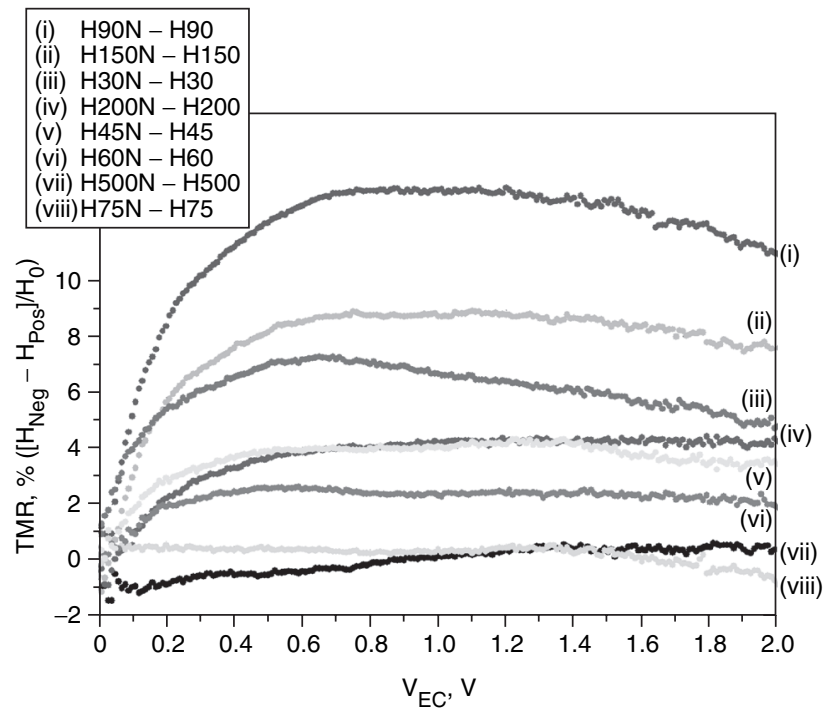

Fig. 36 Calculated TMR of emitter current for the p-type spin diffusion transistor in common-collector configuration and as a function of both emitter-collector voltage and applied magnetic field at $I_{B}=-0.2 \mu \mathrm{A}$

partially destroy the spin polarisation of the carriers. However, $\mathrm{Al}_{2} \mathrm{O}_{3}$ needs significant work as the deposition of $\mathrm{Al}$ directly onto Si results in the formation of AlSi. As was shown by Schmidt et al. [27], a metal-semiconductor contact will destroy the spin polarisation. It may also increase the contact resistance.

- Implementing different magnetic materials on the collector and emitter: There is insufficient magnetic switching differential (where each contact switches quickly at a particular field) between the three contacts. Furthermore, making the contacts small enough that they are monodomain will sharpen up the magnetic switching behaviour of the device.

- Existence of a contact resistance: The existence of this ohmic contribution limits the output current of the device, thereby reducing the magnitude of current gain the device can produce, as well as affecting the magnetic characteristics. Further investigation on the fabrication of tunnel barriers on Si should remove this.

- Nonoptimal electrode geometry: Recent modelling [28-30] indicates that the emitter efficiency can be dramatically improved by implementing spin injection into a base with length (distance between the collector and base contacts) much greater than the thickness (distance between the emitter and collector contacts) and also by interposing the collector between the emitter and base contact. Tuning of the barrier resistances to optimise conduction in the region of positive current gain is necessary.

- Nonoptimal doping profile: The doping profile in these spin diffusion transistors is not optimal (see Fig. 14). The dopant changes sign in the middle of the silicon base, which creates a weak $\mathrm{p}-\mathrm{n}$ junction that in turn dilutes the spin polarisation as the carriers cross it. This in turn reduces the maximum theoretical magnetic sensitivity.

- The presence of Lorentz MR: This is an intrinsic property of the silicon, but its magnitude may be reduced by careful device geometry. Furthermore, its effect will be rendered less significant by improvement in the spin-selective signal.

Finally, further work needs to be done to improve tunnel barrier fabrication on $\mathrm{Si}$, to determine the correlation 
between deposition parameters, $\mathrm{Si}$ doping and barrier resistance. Preliminary results can be found in [31, 32].

\section{Acknowledgments}

The authors thank the silicon processing team at the University of Southampton, M. Johnson and M. Hehn, for useful discussions, and the EPSRC (GR/L68643/01) and the EC contract SPINOSA (IST-2001-33334) for financial support.

\section{References}

1 Baibich, M.N., Broto, J.M., Fert, A., Dau, F.N.V., Petroff, F., Eitenne, P., Creuzet, G., Friederich, A., and Chazelas, J.: 'Giant magnetoresistance of $(001) \mathrm{Fe} /(001) \mathrm{Cr}$ magnetic superlattices', Phys. Rev. Lett., 1988, 61, pp. 2472-2475

2 Johnson, M.: 'Bipolar spin switch', Science., 1993, 260, p. 320

3 Monsma, D.J., Lodder, J.C., and Popma, T.J.A. et al.: 'Perpendicular hot electron spin-valve effect in a new magnetic field sensor: the spinvalve transistor', Phys. Rev. Lett., 1995, 74, p. 5260

4 Sato, R., and Mizushima, K.: 'Spin-valve transistor with an $\mathrm{Fe} / \mathrm{Au}$ / $\mathrm{Fe}(001)$ base', Appl. Phys. Lett., 2001, 79, p. 1157

5 van Dijken, S., Jiang, X., and Parkin, S.S.P.: 'Room temperature operation of a high output current magnetic tunnel transistor', Appl. Phys. Lett., 2002, 80, p. 3364

6 Ounadjela, K., and Hehn, M.: Patent Application Number FR9904227 (France, 2000)

7 Datta, S., and Das, B.: 'Electronic analog of the electro-optic modulator', Appl. Phys. Lett., 1990, 56, p. 665

8 Fabian, J., Zŭtić, I., and das Sarma, S.: 'Magnetic bipolar transistor', Appl. Phys. Lett., 2004, 84, p. 85

9 Fabian, J., and Zŭtić, I.: 'Spin-polarized current amplification and spin injection in magnetic bipolar transistors', Phys. Rev. B, Condens. Matter Mater. Phys., 2004, 69, p. 115314

10 Johnson, M.: 'The all-metal spin transistor', IEEE Spectrum, 1994, 31, (5), p. 47

11 Gardelis, S., Smith, C.G., and Barnes, C.H.W. et al.: 'Spin-valve effects in a semiconductor field-effect transistor: A spintronic device', Phys. Rev. B, Condens. Matter., 1999, 60, p. 7764

12 Private communication, cited in [8]

13 Bandyopadhyay, S., Cahay, M.: 'Are spin junction transistors suitable for signal processing?' Appl. Phys. Lett., 2005, 86, p. 133502

14 Mead, C.A.: 'The tunnel-emission amplifier', Proc. IRE., 1960, 48, pp. 359-361
15 Kisaki, H.: 'Tunnel transistor', Proc. IEEE., 1973, 61, pp. 1053-1054

16 Gregg, J.F., and Sparks, P.D.: Patent Application Number US6218718, (USA, 2001)

17 Dennis, C.L., Siristhatikul, C., Ensell, G.J., Gregg, J.F., and Thompson, S.M.: 'A high-current gain silicon-based spin transistor', J. Phys. D, Appl. Phys., 2003, 36, pp. 81-87

18 Dennis, C.L.: 'A Silicon-based spin transistor'. PhD thesis, the University of Oxford, 2004

19 Mott, N.F.: 'Electrons in disordered structures', Adv. Phys., 2004, 50, pp. $865-945$

20 Xu, Y., Ephron, D., and Beasley, M.R.: 'Directed inelastic hopping of electrons through metal-insulator-metal tunnel junctions', Phys. Rev. B, Condens. Matter., 1995, 52, p. 2843

21 Fowler, R.H., and Nordheim, L.W.: 'Electron emission in intense electric fields', Proc. R. Soc. Lond A, 1928, 119, p. 173

22 Manoharan, S.S., Elefant, D., Reiss, G., and Goodenough, J.B.: 'Extrinsic giant magnetoresistance in chromium (IV) oxide, $\mathrm{CrO} 2$ ', Appl. Phys. Lett., 1998, 72, p. 984

23 Schwerer, F.C., and Silcox, J.: 'Electrical resistivity of nickel at low temperatures', Phys. Rev. Lett., 1968, 20, p. 101

24 Dennis, C.L., Gregg, J.F., Thompson, S.M., and Ensell, G.J.: 'Evidence for electrical spin tunnel injection into Si', submitted 2004

25 Julliere, M.: 'Tunneling between ferromagnetic films', Phys. Lett., 1975, 54A, p. 225

26 Coey, J.M.D.: 'Materials for spin electronics', in Thornton, M. and Ziese, M.: (Eds.).: 'Spin electronics' (Springer, New York, 2001), p. 278

27 Schmidt, G., Ferrand, D., Molenkamp, L.W., Filip, A.T., and van Wees, B.J.: 'Fundamental obstacle for electrical spin injection from a ferromagnetic metal into a diffusive semiconductor', Phys. Rev. B, Condens. Matter., 2000, 62, p. R4790

28 Borges, R.P., Dennis, C.L., Gregg, J.F., Jouguelet, E., Ounadjela, K. Petej, I., Thompson, S.M., and Thornton, M.J.: 'Comparative study of spin injection into metals and semiconductors', J. Phys. D, Appl. Phys., 2002, 35, pp. 186-191

29 Gregg, J.F., Borges, R.P., Jouguelet, E., Dennis, C.L., Petej, I. Thompson, S.M., and Ounadjela, K.: 'Spin injection efficiency in spin electronic devices', J. Magn. Magn. Mater., 2003, 265, pp. 274 287

30 Dennis, C.L., Tiusan, C.V., Gregg, J.F., Thompson, S.M., Ensell, G.J.: 'Characteristics of Si-based spin tunnel transistor: theory and experiment', in preparation, 2005

31 Dennis, C.L., Tiusan, C.V., Ferreira, R.A., Gregg, J.F., Ensell, G.J., Thompson, S.M., and Freitas, P.P.: 'Tunnel barrier fabrication on $\mathrm{Si}$ and its impact on a spin transistor', J. Magn. Magn. Mater., 2005, 1383, pp. 290-291

32 Dennis, C.L., Ferreira, R.A., Tiusan, C.V., Gregg, J.F., and Freitas, P.P.: 'Tunnel barrier fabrication on $\mathrm{Si}$ and its application to spintronics', in preparation, 2005 